\title{
Whitney towers and abelian invariants of knots
}

\author{
Jae Choon Cha ${ }^{1,2} \cdot$ Kent E. Orr ${ }^{3} \cdot$ Mark Powell $^{4}$
}

Received: 16 August 2016 / Accepted: 4 February 2019 / Published online: 5 April 2019

(c) The Author(s) 2019

\begin{abstract}
We relate certain abelian invariants of a knot, namely the Alexander polynomial, the Blanchfield form, and the Arf invariant, to intersection data of a Whitney tower in the 4-ball bounded by the knot. We also give a new 3-dimensional algorithm for computing these invariants.
\end{abstract}

Keywords Whitney towers · Alexander polynomial · Arf invariant $\cdot$ Blanchfield form

Mathematics Subject Classification 57M25 · 57M27 · 57N13 · 57N70

\section{Introduction}

We show that intersection data in Whitney towers determines abelian invariants of knots, particularly the Blanchfield form, the Alexander polynomial, and the Arf invariant.

Briefly speaking, a Whitney tower traces an iterated attempt to alter an immersed disc in a 4-manifold to an embedded disc by Whitney moves. Whitney towers naturally approximate an embedded disc. In particular, since the work of Cochran et al. [5], Whitney towers in 4-space have been commonly used to measure the degree to which a knot fails to be slice.

Our main result algorithmically computes the Blanchfield form and the Alexander polynomial of a knot using intersection data from an order two twisted Whitney tower in the 4-disc bounded by the knot. This relates two incarnations of the Arf invariant of a knot using

Jae Choon Cha was partially supported by NRF Grants 2013067043 and 2013053914. Kent E. Orr is supported by Simons Foundation Grant \# 430351. Mark Powell was supported by an NSERC Discovery Grant.

$\bowtie$ Mark Powell

mark.a.powell@durham.ac.uk

Jae Choon Cha

jccha@postech.ac.kr

Kent E. Orr

korr@indiana.edu

1 Department of Mathematics, POSTECH, Pohang, Gyeongbuk 37673, Republic of Korea

2 School of Mathematics, Korea Institute for Advanced Study, Seoul 02455, Republic of Korea

3 Department of Mathematics, Indiana University, Bloomington, IN 47405, USA

4 Department of Mathematical Sciences, Durham University, Durham, UK 
a 4-dimensional argument-one characterizing the Arf invariant in terms of Whitney towers, the other in terms of the Alexander polynomial.

\subsection{Intersection data from order two towers and abelian invariants}

The Seifert pairing provides a well-known method to compute a presentation for the Alexander module of a knot [23]. As a bonus, one easily computes the Alexander polynomial and the Arf invariant. The Seifert pairing also gives rise to a formula for the Blanchfield form of the knot $[12,15]$. See also [10].

This paper takes a different approach, replacing the Seifert surface with a Whitney tower in the 4-disc. This approach promises many advantages, among these that higher order Whitney towers may present modules corresponding to nilpotent and solvable covers of the knot.

Every knot $K \subset S^{3}$ bounds an order two Whitney tower in $D^{4}$, as we demonstrate in Sect. 2. Recall that this means $K$ is the boundary of an immersed (order 0) disc, $D_{0} \rightarrow D^{4}$, with $d=2 k$ self-intersections occurring in oppositely signed pairs. Immersed Whitney discs (of order 1), $D_{1}^{1} \cup \cdots \cup D_{1}^{k}$, arise from each of $k$ cancelling pairs of intersection points. Furthermore, (order two) discs pair order one intersections, which are intersections between order 0 and order 1 discs. That is, an order two Whitney tower is built from immersed Whitney discs which pair all intersections of order less than 2 in the tower.

In a neighbourhood of each intersection point, two local discs, called sheets, intersect transversely. We will see that an order 2 Whitney tower can be improved as follows:

(i) $\pi_{1}\left(D^{4} \backslash v D_{0}\right) \cong \mathbb{Z}$;

(ii) $D_{0} \cap$ int $D_{1}^{j}=\emptyset$ for each $j$; that is, the tower has no order 1 intersections and thus has no order 2 discs.

(iii) For each disc, $D_{1}^{j}$, we can choose one of the two associated double points. This double point comes with an immersed $\operatorname{disc} A_{1}^{j}$ in $D^{4} \backslash \nu D_{0}$ bounded by a loop leaving the double point along one sheet of the intersection and returning to the double point along the other sheet; $A_{i}^{j}$ is called an accessory disc.

We remark that we do not impose any framing conditions on the $D_{1}^{j}$ nor on the $A_{1}^{j}$. Experts will know how to construct such a Whitney tower, but we include a complete proof in Sect. 2.

Definition 1.1 An order two Whitney tower equipped with accessory discs, namely $D_{0} \cup$ $\left(\bigcup_{j} D_{1}^{j}\right) \cup\left(\cup_{j} A_{1}^{j}\right)$, is an order two presentation tower for $K$ if the conditions above are satisfied.

We will view such a tower as a geometric analogue of a presentation matrix for the Alexander module, one which packages the abelian invariants we study.

Now we describe such a presentation matrix, arising from the intersection data of the discs in a presentation tower. Define $W:=D^{4} \backslash v D_{0}$ to be the exterior of the order zero disc. The intersection pairing of transverse 2-chains in $W$ takes values in the group ring $\mathbb{Z}\left[\pi_{1}(W)\right]=\mathbb{Z}[\mathbb{Z}] \cong \mathbb{Z}\left[t, t^{-1}\right]$. Let $e_{2 i-1}=D_{1}^{i}$ and $e_{2 i}=A_{1}^{i}$. Let $\Lambda=\left(\lambda_{i j}\right)$ be the $d \times d$ matrix over $\mathbb{Z}\left[t, t^{-1}\right]$ whose $(i, j)$-entry, $\lambda_{i j}$, is the $\mathbb{Z}\left[t, t^{-1}\right]$-valued intersection of $e_{i}$ and $e_{j}$. To define the diagonal entry $\lambda_{i i}$, which is the intersection of $e_{i}$ and a push-off of $e_{i}$, we need a section of the normal bundle of the (Whitney or accessory) disc, along which the push-off is taken. For this purpose we use an extension of the Whitney framing and accessory framing of the boundary of the disc. A detailed description is given in Sects. 3 and 4.3. For now we remark that the twisting information of the order one Whitney discs and accessory discs is reflected in these diagonal matrix entries. 
Now let $E=\left(\varepsilon_{i j}\right)$ be the $d \times d$ matrix given by

$$
\varepsilon_{i j}= \begin{cases}\text { the sign of } p & \begin{array}{l}
\text { if } i=j \text { and } e_{i} \text { is an accessory disc based at a double point } p \\
1
\end{array} \\
\text { if one of } e_{i} \text { and } e_{j} \text { is an accessory disc for a double point } p \\
\text { and the other is a Whitney disc with } p \text { on the boundary, } \\
\text { otherwise. }\end{cases}
$$

Define $\Omega:=z \Lambda+E$ where $z:=(1-t)\left(1-t^{-1}\right)$. We say that two polynomials in $\mathbb{Z}\left[t, t^{-1}\right]$ are equal up to norms and units if they agree in the quotient of $\mathbb{Z}\left[t, t^{-1}\right]$ by the multiplicative subgroup

$$
\left\{ \pm t^{k} f(t) f\left(t^{-1}\right)\left|k \in \mathbb{Z}, f(t) \in \mathbb{Z}\left[t, t^{-1}\right],\right| f(1) \mid=1\right\} .
$$

In the following theorem we show that the matrix $\Omega$ presents the Blanchfield pairing (see Definition 10.1) up to Witt equivalence, and thus determines the Alexander polynomial up to norms and units.

Theorem 1.2 The matrix $\Omega$ is a presentation matrix for a linking form Witt equivalent to the Blanchfield form of $K$. The determinant of $\Omega$ equals the Alexander polynomial of $K, \Delta_{K}(t)$, up to norms and units.

A variation on the above theorem arises by replacing the Whitney discs with additional accessory discs in the following way. Recall that the Whitney disc $e_{2 i-1}=D_{1}^{i}$ joins two selfintersection points of $D_{0}$, say $p_{i}$ and $q_{i}$, and the corresponding accessory disc $e_{2 i}=A_{1}^{i}$ is based at one of these, say $p_{i}$. Let $e_{2 i-1}$ be an accessory disc for the other intersection point $q_{i}$. Replace an arbitrary sub-collection of the Whitney discs by accessory discs as above. We obtain an intersection matrix via the same prescription given above and the conclusions of Theorem 1.2 still hold. In particular, Theorem 1.2 holds even when all the order one discs are accessory discs. More precisely, let $e_{i}$ be an accessory disc for the $i$ th double point of $D_{0}, i=1, \ldots, d$. Let $\Lambda=\left(\lambda_{i j}\right)$ where $\Lambda_{i j}$ is the $\mathbb{Z}[\mathbb{Z}]$-intersection number of $e_{i}$ and $e_{j}$. Let $E$ be the $d \times d$ diagonal matrix whose $i$ th diagonal entry is the sign of the $i$ th double point. Define $\Psi=z \Lambda+E$.

Theorem 1.3 The matrix $\Psi$ is a presentation matrix for a linking form Witt equivalent to the Blanchfield form of $K$. The determinant of $\Psi$ equals $\Delta_{K}(t)$, the Alexander polynomial of $K$, up to norms and units.

\section{An algorithm to compute abelian invariants}

Here is a special case of the type of tower used to determine the matrix $\Psi$ in Theorem 1.3. Construct an immersed disc bounded by a knot $K$ as follows. Start with a collection of crossings on a planar diagram of $K$ such that changing these crossings gives the trivial knot. The associated homotopy traces out a level preserving immersed annulus in $S^{1} \times I \hookrightarrow S^{3} \times I$ bounded by $K=K \times\{0\} \subset S^{3} \times\{0\}$, and a trivial knot in $S^{3} \times\{1\}$, whose intersections correspond to the crossing changes. Cap off $S^{3} \times I$ by gluing a copy of $D^{4}$ to $S^{3} \times\{1\}$ and cap off the annulus along its unknotted boundary component to obtain an immersed disc $D_{0}$ in $D^{4}$, which $K$ bounds. Choose an accessory disc for each self-intersection of $D_{0}$. Define $\Psi$ as in Theorem 1.3.

The next result enables us, in this special case, to compute abelian invariants from the intersection data of the immersed tower without the indeterminacy from Witt equivalence and norms. 
Theorem 1.4 The matrix $\Psi$ is a presentation matrix for the Blanchfield form of $K$. In particular, the determinant of $\Psi$ equals $\Delta_{K}(t)$ up multiplication by a unit $\pm t^{k}$.

In addition, we show that for a special choice of accessory discs, the computation of the intersection data (and consequently of the abelian invariants) is algorithmic from a knot diagram, providing a new 3-dimensional procedure to compute the Alexander polynomial and the Blanchfield form of a knot. We describe the algorithm in Sect. 7.2, and we work through a detailed example in Sect. 7.3.

\subsection{Whitney towers and the Arf invariant}

Recall that we used the Whitney framing to compute the $\mathbb{Z}\left[t, t^{-1}\right]$-valued intersection number of an order one Whitney disc $D_{1}^{i}$ with itself. In general, an extension of the Whitney framing to $D_{1}^{i}$ may have zeros; the Whitney framing extends to a non-vanishing section on $D_{1}^{i}$ if and only if it agrees with the unique framing of the normal bundle of $D_{1}^{i}$. Following common convention, we call such a Whitney disc framed. A Whitney tower is framed if all the Whitney discs in the tower are framed.

The generic number of zeroes, counted with sign, of an extension of the Whitney framing to the normal bundle of the Whitney disc is called the twisting coefficient. If a given Whitney disc is not framed, by interior twisting we can alter the twisting coefficient by any multiple of 2 , and whence if the twisting coefficient were even, we could arrange that the Whitney disc be framed. This motivates the following definition.

Definition 1.5 A Whitney disc is essentially twisted if its twisting coefficient is odd.

We recall the definition of the Arf invariant of a knot, in terms of a Seifert matrix, in Definition 8.2. The following theorem follows from work of Matsumoto, Kirby, Freedman and Quinn [8,17], [9, Section 10.8]. See also [7, Lemma 10].

Theorem 1.6 (Freedman, Kirby, Matsumoto, Quinn) The Arf invariant $\operatorname{Arf}(K)$ vanishes if and only if $K$ is the boundary of framed Whitney tower of order two in $D^{4}$.

In fact, Schneiderman [21] also showed that the Arf invariant is the only obstruction for a knot to bound a framed (asymmetric) Whitney tower of any given order: a knot which bounds a framed order two Whitney tower in $D^{4}$ bounds a framed order $n$ Whitney tower for all $n$.

Levine showed that the Arf invariant of a knot, defined in terms of the Seifert form (recalled in Definition 8.2), can be computed in terms of the Alexander polynomial $\Delta_{K}(t)$ [14, Sections 3.4 and 3.5]. He used the fact that the Alexander polynomial can be computed as $\operatorname{det}\left(t V-V^{T}\right)$, where $V$ is a Seifert matrix for $K$.

Theorem 1.7 (Levine) The Arf invariant $\operatorname{Arf}(K)$ of a knot $K$ satisfies:

$$
\operatorname{Arf}(K)=\left\{\begin{array}{lll}
0 & \text { if } \Delta_{K}(-1)= \pm 1 & \bmod 8 \\
1 & \text { if } \Delta_{K}(-1)= \pm 3 & \bmod 8
\end{array}\right.
$$

The absolute value of the Alexander polynomial evaluated at -1 is also the order of the homology of the twofold branched cover of $K$, which is a $\mathbb{Z}_{(2)}$-homology circle. In particular, $\Delta_{K}(-1)$ is always an odd number. The Arf invariant measures, up to a unit, whether $\Delta_{K}(-1)$ is a square modulo 8 .

By combining the two previous theorems, the following is known. 
Theorem 1.8 (Freedman, Kirby, Levine, Matsumoto, Quinn) A knot $K$ bounds a framed Whitey tower of order two if and only if $\Delta_{K}(-1) \equiv \pm 1 \bmod 8$.

However the only previously known proof of this result (to the authors) proceeds by passing via the Seifert form definition of the Arf invariant. We give a new, direct, 4-dimensional proof that the Whitney tower and Alexander polynomial interpretations of the Arf invariant are equivalent. More precisely, we show the following.

Theorem 1.9 Suppose $K$ bounds an order two Whitney tower where $n$ of the order one Whitney discs are essentially twisted. Then

$$
\Delta_{K}(-1) \equiv\left\{\begin{array}{llll} 
\pm 1 & \bmod 8 & \text { if } n \equiv 0 & \bmod 2 \\
\pm 3 & \bmod 8 & \text { if } n \equiv 1 & \bmod 2
\end{array}\right.
$$

If an order 2 Whitney tower has an even number of essentially twisted Whitney discs, then it can be modified by geometric moves to a framed order 2 tower. This follows easily from [6, Theorem 2.15]; for the convenience of the reader we sketch the procedure in Lemma 2.4. However note that we do not need this step: the Alexander polynomial conclusion can be drawn if we have an order two Whitney tower with an even number of essentially twisted discs.

\section{Motivation}

In future work, we hope to describe all nilpotent invariants of links (roughly, invariants carried by duality and the homology of a nilpotent cover) from the intersection theory of an asymmetric Whitney tower for the link.

Of particular interest are the postulated "higher order Arf invariants" of Conant et al. [6,7]. They ask whether a link bounds an asymmetric framed Whitney tower in the 4-ball, and define an obstruction theory involving an algebra of labelled uni-trivalent trees. They show that Milnor's link invariants and the Arf invariant are obstructions to building towers. Additional non-trivial trees in their algebra do not correspond to any known invariants, and may obstruct higher order framed Whitney towers for certain links. The main examples of these links are iterated Bing doubles of knots with non-vanishing Arf invariant. Conant, Schneiderman and Teichner call these invariants the higher order Arf invariants, and these invariants live either in $\mathbb{Z}_{2}$ or 0 . If the higher order Arf invariants were trivial, one would need to add new relations to the tree algebra. We recommend [6,7] for further reading. It is with this problem in mind that we put such emphasis on giving a new proof of the long-known relationship between Whitney towers and the Arf invariant.

\section{Organisation of the paper}

Section 2 constructs a presentation tower for the knot, that is an order two immersed Whitney tower with the special attributes described in Definition 1.1. Section 3 gives the statement of our main technical theorems, Theorems 3.1 and 3.2, on the structure of the intersection form of the exterior $W$ of an immersed disc $D_{0} \rightarrow D^{4}$, and the relation of this intersection form to the combinatorics of Whitney and accessory disc intersections. Section 4 is devoted to the proof of the technical theorems. Section 4.1 shows that $\pi_{2}(W)$ is a free module. Section 4.2 constructs the spheres we use to compute the intersection form. Section 4.3 gives the precise definitions of Whitney and accessory framings. Sections 4.4 through 4.9 
compute the intersections of the spheres, proving Theorems 3.1 and 3.2. Section 5 computes the homology of $\partial W$. Section 6 collates the results of the previous two sections, proving Theorems 1.2, 1.3 and 1.4, apart from the Blanchfield form assertions. Section 7 gives some example computations. Section 8 recalls, for completeness, the usual definition of the Arf invariant in terms of the Seifert form. Section 9 proves Theorem 1.9 relating the Alexander polynomial at -1 to the modulo two count of the number of twisted Whitney discs. Section 10 considers the Blanchfield form and completes the proof of Theorems 1.3 and 1.2.

\section{Construction of an order two presentation tower for a knot}

We begin with a properly immersed disc $D_{0}^{\prime}$ in $D^{4}$ with boundary a knot $K \subset S^{3}$ which has an algebraically vanishing count of self-intersection points. This can always be arranged by adding local cusp singularities to $D_{0}^{\prime}[13, \mathrm{p}$. 72]. Such a disc induces the zero framing on its boundary $K$. In the next two subsections, we will show how to find a new immersed disc $D_{0}$, regularly homotopic to $D_{0}^{\prime}$, the complement of which has infinite cyclic fundamental group. We will then show how to find order one Whitney discs $D_{1}^{1}, \ldots, D_{1}^{k}$, that are potentially twisted, in the exterior of $D_{0}$. Here $D_{0}$ has $d=2 k$ double points. In our results relating knot invariants to Whitney towers, we will use intersection data from the order one Whitney discs, together with data from additional discs called accessory discs. This will construct an order two presentation tower for $K$, as promised.

For a double point $p$ of $D_{0}$, a double point loop is a loop on $D_{0}$ that leaves $p$ along one sheet and returns along the other, avoiding all other intersection points. An accessory disc (see [9, Section 3.1]) is a disc in $D^{4} \backslash \nu D_{0}$ whose boundary is a push-off of a double point loop to the boundary $\partial_{+}:=\partial\left(\operatorname{cl}\left(v D_{0}\right)\right) \backslash v K$ of a neighbourhood of $D_{0}$. By a judicious choice, the push-off can be arranged to be trivial in $\pi_{1}\left(D^{4} \backslash \nu D_{0}\right) \cong \mathbb{Z}$. It therefore bounds an accessory disc in $D^{4} \backslash v D_{0}$. (See Lemma 2.2 below.)

For each Whitney disc $D_{1}^{i}$, pick one of the two intersections paired by $D_{1}^{i}$, and produce an accessory disc $A_{1}^{i}$ for this intersection as above.

\subsection{Fixing the fundamental group}

Lemma 2.1 A properly immersed disc $D_{0}^{\prime}$ in $D^{4}$ with boundary a knot $K \subset S^{3}$ is regularly homotopic to a disc $D_{0}$ for which $\pi_{1}\left(D^{4} \backslash \nu D_{0}\right) \cong \mathbb{Z}$. Moreover, new double points support order 1 framed Whitney discs.

Proof The idea is to use finger moves, as introduced by Casson [3]. A finger move kills a commutator of the form $\left[g, g^{w}\right]$, where $g$ is a meridian of $D_{0}^{\prime}, w$ is the curve the finger pushes along, and $g^{w}$ means $w g w^{-1}$.

Apply finger moves to make any pair of meridional loops commute. Since meridional loops (finitely) generate the fundamental group, the fundamental group $\pi_{1}\left(D^{4} \backslash v D_{0}\right)$ corresponding to the new immersed disc $D_{0}$ is the abelianisation of $\pi_{1}\left(D^{4} \backslash v D_{0}^{\prime}\right)$ which is $\mathbb{Z}$.

Define $W:=D^{4} \backslash \nu D_{0}$ to be the exterior of the immersed disc $D_{0}$ produced by Lemma 2.1 . A consequence of Lemma 2.1 is the existence of an accessory disc.

Lemma 2.2 Each double point of $D_{0}$ has an accessory disc in $W$.

Proof Choose a push-off of a double point loop. By winding the push-off around a meridian to $D_{0}$ if necessary, arrange that the push-off is null-homotopic in $W$. Here we use that 
$\pi_{1}(W) \cong \mathbb{Z}$. A null-homotopy in general position gives us an accessory disc as required. Here we do not impose any framing condition on the accessory disc.

The same argument applies to the Whitney disc case, showing that any pair of double points with opposite sign admit a (potentially twisted) order one Whitney disc in $W$.

\subsection{Arranging $D_{0} \cap D_{1}=\emptyset$}

A Whitney tower of order one is a properly immersed disc $D_{0}$ together with Whitney discs $D_{1}=D_{1}^{1} \cup \cdots \cup D_{1}^{k}$ which pair up all the double points of $D_{0}$. The Whitney discs are said to have order one (since they pair self-intersections of the order zero disc.) We impose nothing about the framing of the Whitney discs. We remark that we can indeed arrange each Whitney disc to be framed, by applying boundary twists, and in this case the tower is called a framed Whitney tower of order one.

In an order one Whitney tower, since a Whitney disc pairs double points of opposite signs, $D_{0}$ automatically has vanishing algebraic self intersection. Conversely, when $D_{0}$ is an immersed disc in $D^{4}$ with algebraic self-intersection zero then, since $D^{4}$ is simply connected, there exist Whitney discs which pair up all the double points.

Furthermore, for any given order one Whitney tower, we can modify the tower so that the interiors of the order one Whitney discs are disjoint from the order zero disc $D_{0}$, as required in the definition of an order 2 presentation tower (Definition 1.1). For the convenience of the reader, we explain the procedure in the next lemma, which is well known to the experts.

This is a special case of a general result of Conant et al. c.f. [7, Proof of Lemma 10]. However, note that Conant et al. do not need to actually cancel intersection points geometrically; in their situation it is enough to pair them up with Whitney discs which admit higher order intersections only. For this reason we spell out the details in our special case. If one wishes to simply show the existence of an order two presentation tower, rather than promoting a given order one Whitney tower, one can choose Whitney discs in the exterior of $D_{0}$, as in the remark just after the proof of Lemma 2.2.

Everything in 4-manifold topology seems to comes at a price, and in this case we can arrange the desired disjointness $D_{0} \cap D_{1}=\emptyset$ at the cost of allowing twisted Whitney discs.

Lemma 2.3 Let $D_{0} \cup D_{1}$ be an order one Whitney tower, where $D_{1}=D_{1}^{1} \cup \cdots \cup D_{1}^{n}$. After performing boundary twists on $D_{1}$, there is a regular homotopy of $D_{0}$ to an immersed disc $D_{0}^{\prime}$ which supports an order 2 tower $D_{0}^{\prime} \cup D_{1}^{\prime}$ where $D_{0}^{\prime} \cap$ int $D_{1}^{\prime}=\emptyset$.

Proof A boundary twist [9, Section 1.3] of an order one Whitney disc $D_{1}^{i}$ adds an intersection point $D_{1}^{i} \cap D_{0}$. Perform boundary twists until all such intersection points occur algebraically zero times. The $D_{1}^{i}$ may now be twisted (essentially or otherwise). Pair up the intersection points in $D_{0} \cap D_{1}^{i}$ and find Whitney discs $D_{2}$ for each pair. These always exist by simple connectivity of $D^{4}$. However we may have that $D_{1} \cap D_{2}$ and $D_{0} \cap D_{2}$ are nonempty. Push the intersections $D_{1} \cap D_{2}$ off $D_{2}$ over the $D_{1}$ part of its boundary by a finger move. This creates new $D_{1} \cap D_{1}$ intersections but we do not mind. Push the intersections $D_{0} \cap D_{2}$ off the $D_{0}$ part of the boundary. This creates new $D_{0} \cap D_{0}$ intersections. These have to be paired up with a new order 1 Whitney disc $D_{1}^{j}$. This is always possible, since the new intersections came from a finger move (note that the new disc $D_{1}^{j}$ is framed). One has to be careful that the new Whitney arcs for the new $D_{1}^{j}$ do not intersect the Whitney $\operatorname{arcs}$ for $D_{2}$. This can easily be arranged by pushing the boundary arc (see [20, Figures 6,7 and 8]), but means that the new $D_{1}^{j}$ intersects the old $D_{1}^{i}$ (the order one disc whose intersections with $D_{0}$ are 
being paired up by $D_{2}$ ). However new $D_{1} \cap D_{1}$ intersections are allowed. We have now arranged that $D_{2}$ is disjoint from everything. Therefore we can use it to perform the Whitney move. Push $D_{1}^{i}$ across $D_{2}$. Any self-intersections of $D_{2}$ result in more $D_{1} \cap D_{1}$ intersections, but again these are permitted. We have now decreased the number of intersection points in $D_{0} \cap D_{1}$ by two, at the cost of new intersection points in $D_{0} \cap D_{0}, D_{1} \cap D_{1}$, potentially twisting a $D_{1}$ disc, and a new $D_{1}$ Whitney disc which is disjoint from $D_{0}$. These are all within our budget. By repeating this process we can therefore arrange that $D_{1} \cap D_{0}=\varnothing$ as claimed. All the operations apart from the boundary twists are regular homotopies on the original discs, together with introducing new order 1 Whitney discs to pair up new $D_{0} \cap D_{0}$ intersections.

We quickly indicate how to see the following statement, since the argument of the proof of Lemma 2.3 is pertinent. We do not need the following lemma but include it for completeness, since it is closely related to Theorem 1.9.

Lemma 2.4 Let $D_{0} \cup D_{1}$ be an order two Whitney tower with an even number of essentially twisted Whitney discs and $D_{0} \cap$ int $D_{1}=\emptyset$. Then there is a regular homotopy of $D_{0}$ to a new immersed disc $D_{0}^{\prime}$ which supports a framed Whitey tower of order two $D_{0}^{\prime} \cup D_{1}^{\prime}$ with $D_{0}^{\prime} \cap D_{1}^{\prime}=\emptyset$.

Proof For each pair of essentially twisted Whitney discs, perform interior twists so that one has twisting coefficient +1 and the other has twisting coefficient -1 . Then perform boundary twists so that both are framed. This introduces a pair of $D_{1} \cap D_{0}$ intersections. The proof of [6, Theorem 2.15] enables us to perform regular homotopies so that these arise on the same order 1 Whitney disc. We may then pair them up with an order 2 Whitney disc $D_{2}$. Now we apply the argument of the proof of Lemma 2.3 to trade the $D_{0} \cap D_{1}$ intersections for higher order $D_{1} \cap D_{1}$ intersections and potentially new $D_{0} \cap D_{0}$ intersections which are paired by new framed order 1 discs. This produces an order 2 framed Whitney tower as claimed.

\section{The intersection form of an immersed disc exterior in the 4-ball}

In this section we give the detailed description of the matrices $\Omega$ and $\Psi$ from the introduction (Theorems 1.2 and 1.3 respectively), in terms of intersection data of the Whitney and accessory discs, and we state our main technical results, that relate these matrices to the intersection pairing of an immersed disc exterior.

Suppose that a knot $K$ bounds an order two presentation tower as constructed in Sect. 2, where the order zero disc $D_{0}$ has $d=2 k$ self-intersection points. We may assume by Lemma 2.1 that $\pi_{1}\left(D^{4} \backslash v D_{0}\right) \cong \mathbb{Z}$. Consider the free module $\mathbb{Z}[\mathbb{Z}]^{d}$, with basis elements $e_{2 i-1}, i=1, \ldots, k$ corresponding to order one Whitney discs $D_{1}^{1}, \ldots, D_{1}^{k}$ pairing up the double points, and with the basis elements $e_{2 i}, i=1, \ldots, k$ corresponding to accessory discs $A_{1}^{1}, \ldots, A_{1}^{k}$ (see [9, Section 3.1] and Lemma 2.2) for half of the self-intersections of $D_{0}$, the double point with a positive sign for each pair which is paired up by one of the $D_{1}^{i}$.

The matrix $\Omega$ described below is hermitian, that is $\Omega=\bar{\Omega}^{T}$, and defines a pairing $\Omega: \mathbb{Z}[\mathbb{Z}]^{d} \times \mathbb{Z}[\mathbb{Z}]^{d} \rightarrow \mathbb{Z}[\mathbb{Z}]$. Here the overline denotes the involution on the group ring $\mathbb{Z}[\mathbb{Z}]$ defined by extending $t \mapsto t^{-1}$ linearly. We abuse notation and conflate the matrix and the pairing which it determines on $\mathbb{Z}[\mathbb{Z}]^{d}$.

As before define $W:=D^{4} \backslash v D_{0}$. Choose a path from a chosen basepoint of each $D_{i}^{i}$, and of each $A_{1}^{j}$, to the basepoint of $W$. For each intersection point $q$ involving $D_{1}^{i}$, choose a path 
from $q$ to the basepoint of $D_{1}^{i}$, inside $D_{1}^{i}$ and missing all double points. Similarly for the $A_{1}^{i}$. For each intersection point in $D_{1}^{i} \cap D_{1}^{j}, D_{1}^{i} \cap A_{1}^{j}$ and $A_{1}^{i} \cap A_{1}^{j}$, there is an associated element $\pm t^{\ell}$ of $\pi_{1}(W) \cong \mathbb{Z}$, defined by considering the usual concatenation of paths. By summing over such intersection points we obtain an element $p(t)$ of $\mathbb{Z}[\mathbb{Z}]$. Let $p_{r s}(t)$ be the polynomial associated to the pair $\left(e_{r}, e_{s}\right)$. Note that $p_{r s}(t)=\overline{p_{s r}(t)}$. When $i=j$, we abuse notation and use $D_{1}^{i} \cap D_{1}^{i}$ and $A_{1}^{i} \cap A_{1}^{i}$ for the double point set of the immersion. Here there is an indeterminacy in $p_{r r}(t)$, up to $t=t^{-1}$, due to a lack of ordering of sheets at an intersection point. However this will not affect the outcome of the computation, so we may make any choice of ordering.

\subsection{Precise description of the matrix $\Omega$}

The $(r, s)$-entry $\Omega_{r s}$ corresponds to intersection data involving the discs associated to the pair $\left(e_{r}, e_{s}\right)$ as given below. The order of the pair matters since $\Omega_{r s}=\bar{\Omega}_{s r}$. Define $z:=$ $(1-t)\left(1-t^{-1}\right)$.

- For $r \neq s$ and $\{r, s\} \neq\{2 i-1,2 i\}, \Omega_{r s}=z p_{r s}(t)$.

- For $\{r, s\}=\{2 i-1,2 i\}$ for some $i, \Omega_{r s}=z p_{r s}(t)+1$, where $p_{r s}(t)$ is computed from intersection points $D_{1}^{i} \cap A_{1}^{i}$.

- When $r=s=2 i-1, \Omega_{r s}=z p_{r r}(t)+\overline{z p_{r r}(t)}+z a_{i}$ where $p_{r r}(t)=p_{s s}(t)$ arises from the self intersection points of $D_{1}^{i}$, and $a_{i} \in \mathbb{Z}$ is the twisting of the Whitney framing relative to the disc framing for $D_{1}^{i}$.

- When $r=s=2 i, \Omega_{r s}=z p_{s s}(t)+\overline{z p_{s s}(t)}+z b_{i}+1$ where $p_{s s}(t)=p_{r r}(t)$ arises from the self intersection points of $A_{1}^{i}$, and $b_{i} \in \mathbb{Z}$ is the twisting of the accessory framing relative to the disc framing for $A_{1}^{i}$.

The first and last cases only are relevant to $\Psi$ from Theorem 1.3. Precise definitions of the Whitney and accessory framings are given in Sect. 4.3.

For practical purposes it is not always convenient to have the accessory disc correspond to a double point with positive intersection sign. If we use a double point with negative sign, then replace the +1 in $\Omega_{2 i, 2 i}$ entry in the last bullet point with a -1 .

\subsection{Structure of the intersection form of $W$}

The following is one of our main technical results.

Theorem 3.1 Suppose that $D_{0}$ has $d=2 k$ double points, and $D_{1}^{i}$, for $i=1, \ldots, k$, are order one Whitney discs pairing up the double points of $D_{0}$, whose interiors are disjoint from $D_{0}$. Let $A_{1}^{i}$, for $i=1, \ldots, k$, be an accessory disc for the $2 i$-th double point, where the double points are ordered so that even numbered points have positive sign. Then we have the following:

(1) The homotopy group $\pi_{2}(W)$ is a free $\mathbb{Z}[\mathbb{Z}]$ module of rank $d$.

(2) There is a linearly independent set $\left\{S_{i}\right\}$ of immersed 2-spheres which generate a free submodule $F$ of $\pi_{2}(W)$ of rankd on which the equivariant intersection form $\lambda: F \times F \rightarrow$ $\mathbb{Z}[\mathbb{Z}]$ can be written as $z\left(X+\left(z Y+\overline{z Y}^{T}\right)\right)$ where $X$ is a block diagonal sum of $k$ copies of the form

$$
\left[\begin{array}{cc}
z a_{i} & 1 \\
1 & 1+z b_{i}
\end{array}\right]
$$


with $a_{i}, b_{i} \in \mathbb{Z}$, and $Y$ is an upper triangular $d \times d$ matrix.

(3) The $S_{i}$ form a basis for $\pi_{2}(W) \otimes_{\mathbb{Z}[\mathbb{Z}]} \mathbb{Z}$.

(4) The coefficients $a_{i}, b_{i} \in \mathbb{Z}$ in the ith $2 \times 2$ block diagonals of $X$ are the twisting numbers of the ith Whitney disc $D_{1}^{i}$ and the ith accessory disc $A_{1}^{i}$ respectively.

(5) The coefficients of $Y$ are the $\mathbb{Z}[\mathbb{Z}]$-twisted intersection numbers and self-intersection numbers of the $D_{1}^{i}$ and the $A_{1}^{i}$.

Comparing the matrix $\Omega$ defined above with the matrix of the intersection form of $W$, we have $\lambda=z \Omega$.

The proof of this theorem will take the entire next section. In the course of the proof we explicitly construct immersed 2-spheres $S_{i}$ which represent elements of $\pi_{2}(W)$ and compute the intersection form using these explicit elements and intersections between Whitney discs and accessory discs.

It is quite possible that $F=\pi_{2}(W)$, however we are only able to prove this in the special case that $D_{0}$ arises from crossing changes; see Lemma 6.2.

We have another version which only uses accessory discs, and which is used to deduce Theorem 1.3. For the purpose of deducing Theorem 1.3 we give the explicit statement.

Theorem 3.2 Suppose that $D_{0}$ has $d$ double points and $A^{i}$ are accessory discs $(i=1, \ldots, d)$ whose interiors are disjoint from $D_{0}$. Then we have the following:

(1) The homotopy group $\pi_{2}(W)$ is a free $\mathbb{Z}[\mathbb{Z}]$ module of rank $d$.

(2) There is a linearly independent set $\left\{S_{i}\right\}$ of immersed 2-spheres which generate a free submodule $F$ of $\pi_{2}(W)$ of rankd on which the equivariant intersection form $\lambda: F \times F \rightarrow$ $\mathbb{Z}[\mathbb{Z}]$ can be written as $z\left(X+\left(z Y+\overline{z Y}^{T}\right)\right)$ where $X$ is a diagonal matrix with entries $\pm 1+z b_{i}$, with $b_{i} \in \mathbb{Z}$, and $Y$ is an upper triangular $d \times d$ matrix.

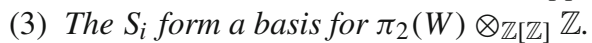

(4) The coefficients $b_{i} \in \mathbb{Z}$ in $X$ are the twisting numbers of the ith accessory disc $A_{i}$, and the \pm 1 is determined by the sign of the ith double point.

(5) The coefficients of $Y$ are the $\mathbb{Z}[\mathbb{Z}]$-twisted intersection numbers and self-intersection numbers of the $A_{i}$.

Compare this with the matrix $\Psi$ from the introduction to observe that $\lambda=z \Psi$. Both sets of spheres from the above two theorems arise from ambient surgery on a basis of $H_{2}(W ; \mathbb{Z})$ comprising Clifford tori of the double points, as we will see in Sect. 4.2. Restricting the proof of Theorem 3.1 to the accessory discs only gives the proof of Theorem 3.2. Therefore we focus on Theorem 3.1.

\section{Proofs of the intersection form Theorems 3.1 and 3.2}

\subsection{The second homotopy group of $W$ is a free module}

In this subsection we prove the following.

Lemma 4.1 The homotopy group $\pi_{2}(W)$ is a free $\mathbb{Z}[\mathbb{Z}]$ module.

Proof Let $R:=\mathbb{Z}\left[\mathbb{Z}\right.$. Since $\pi_{1}(W) \cong \mathbb{Z}$, we have $H_{1}(W ; R)=0$ and $H_{2}(W ; R) \cong$ $\pi_{2}(W)$. We therefore need to show that $H_{2}(W ; R)$ is a free module, which follows from 
general arguments on 4-manifolds with fundamental group $\mathbb{Z}$. The relative cohomology group $H^{2}(W, \partial W ; R)$ can be computed using the universal coefficient spectral sequence

$$
E_{2}^{p, q}=\operatorname{Ext}_{R}^{p}\left(H_{q}(W, \partial W ; R), R\right) \Longrightarrow H^{n}(W, \partial W ; R),
$$

where the differential $d_{r}$ on $E_{r}^{p, q}$ has degree $(r, 1-r)$ (see e.g. [15, Theorem 2.3]). First, from the long exact sequence of a pair and from $H_{1}(W ; R)=0$, it follows that $H_{1}(W, \partial W ; R)=0$. From this and from $H_{0}(W, \partial W ; R)=0$, it follows that the only nontrivial term on the line $p+q=2$ on the $E^{2}$ page is $E_{2}^{0,2}=\operatorname{Hom}_{R}\left(H_{2}(W, \partial W ; R), R\right)$. Since $H_{1}(W, \partial W ; R)=0$ and since $R$ has homological dimension 2 (or since $H_{0}(W, \partial W ; R)=0$ ), the differentials $d_{r}^{0,2}$ at $E_{r}^{0,2}$ for $r \geq 2$ are into trivial codomains and thus trivial. Therefore we deduce that

$$
H^{2}(W, \partial W ; R) \cong \operatorname{Hom}_{R}\left(H_{2}(W, \partial W ; R), R\right) .
$$

This is a free module, since $\operatorname{Hom}_{R}(A, R)$ is free for any $R$-module $A$, by [11, Lemma 3.6] or [2, Lemma 2.1]. Therefore $H^{2}(W, \partial W ; R) \cong H_{2}(W ; R)$ is free as claimed.

\subsection{Construction of spheres in $\pi_{2}(W)$}

We proceed to construct explicit elements of $\pi_{2}(W)$ whose intersection data can be computed in terms of intersection and twisting data for the discs $D_{1}$ and $A_{1}$.

Consider the Clifford torus for a self-intersection point of $D_{0}$. A neighbourhood of a selfintersection point is homeomorphic to $\mathbb{R}^{4}$, in which the two intersecting sheets sit as $\mathbb{R}^{2} \times\{0\}$ and $\{0\} \times \mathbb{R}^{2}$. The Clifford torus $T:=S^{1} \times S^{1} \subset \mathbb{R}^{2} \times \mathbb{R}^{2} \cong \mathbb{R}^{4}$ is shown in a 5-still movie diagram in Fig. 1. We may assume that $T$ lies in $\partial W$. We will call the curves $S^{1} \times *$ and $* \times S^{1}\left(* \in S^{1}\right)$, which are meridians of the two sheets, the standard basis curves of $T$.

We describe the basic construction of a sphere $S_{2 i}$ using an accessory disc $A_{1}^{i}$ for the double point $p$. The authors learnt this construction from Peter Teichner. We will postpone detailed discussion of framing issues for later computations, for now contenting ourselves with conveying the main idea of the construction. We may modify the construction later by inserting interior or boundary twists into the procedure, in order to arrange that our spheres have framed normal bundles.

Consider a double point loop $\alpha$ on $D_{0}$, and consider the normal circle bundle to $D_{0}$ restricted to $\alpha$. This defines a torus in $D^{4}$. The intersection of this torus with $W=D^{4} \backslash v D_{0}$ defines $N:=\left(\alpha \times S^{1}\right) \cap \partial W$, which is the image of the map of an annulus into $W$. The boundary of $N$ is the two generating curves on $T$ for $H_{1}(T ; \mathbb{Z})$. The boundary of $N$ is thus a wedge $S^{1} \vee S^{1}$, since the standard basis curves of $T$ intersect in a single point. The part of $N$ which lies in a $D^{4}$ neighbourhood of the intersection point is shown in Fig. 2.
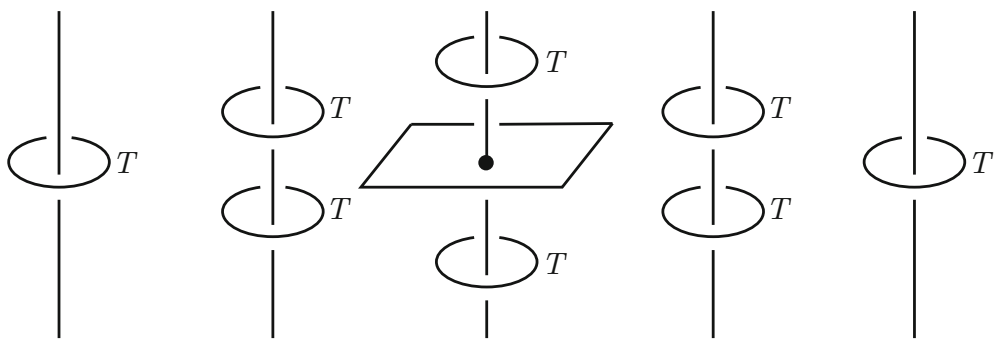

Fig. 1 A Clifford torus $T$ in the neighbourhood of an intersection point of two planes in $\mathbb{R}^{4} \cong \mathbb{R}^{3} \times \mathbb{R}$. One of the planes lies in $\mathbb{R}^{3} \times\{0\}$, while the other intersects each slice $\mathbb{R}^{3} \times\{\mathrm{pt}\}$ in a line 


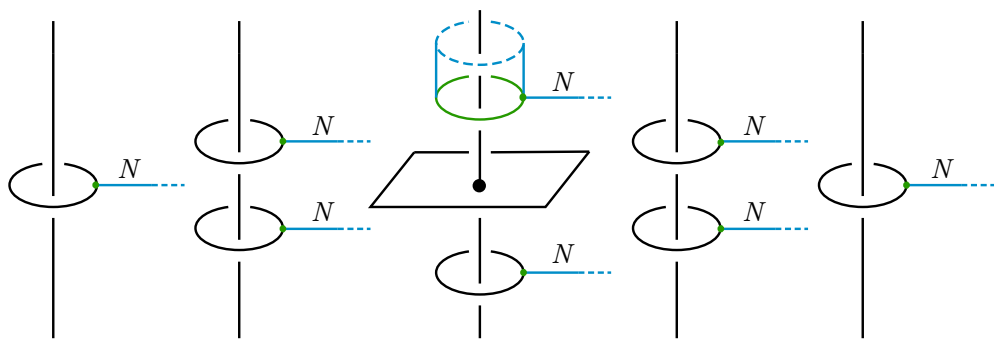

Fig. 2 A Clifford torus $T$ together with the part of the annulus $N$ which lies in a $D^{4}$ neighbourhood of the double point

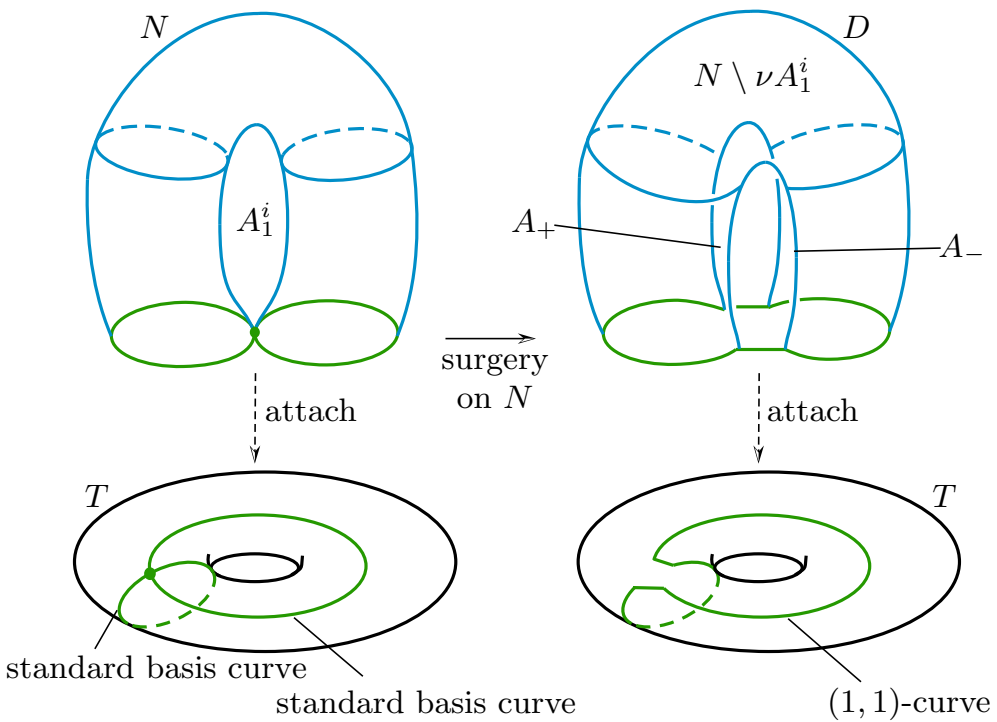

Fig. 3 Surgery on $N$ using $A_{ \pm}$, and the attaching of the resulting disc $D$ to the Clifford torus $T$

We perform a two step ambient surgery process. First use two push-offs of the accessory $\operatorname{disc} A_{1}^{i}$, which we denote by $A_{ \pm}$, to surger $N$ into a disc $D=\left(N \backslash v A_{1}^{i}\right) \cup A_{+} \cup A_{-}$. The boundary of this new disc $D$ is a $(1,1)$ curve on $T$; that is, it represents the sum of a meridian and a longitude in $H_{1}(T ; \mathbb{Z}) \cong \mathbb{Z} \oplus \mathbb{Z}$. In Fig. 3, a schematic of the annulus $N$ is shown, before and after surgery on it has been performed using $A_{+}$and $A_{-}$to convert $N$ into the disc $D$. We also show the attaching of this apparatus to the Clifford torus $T$ in Fig. 3. Next, use two push-offs of $D$ to surger $T$ into an immersed sphere $S_{2 i}$.

For Theorem 3.2 this describes the construction of our entire set of spheres $\left\{S_{i}\right\}$. For Theorem 3.1, this creates half of our spheres: use this construction to produce a sphere from the Clifford torus of one double point in each pair which is paired up by a Whitney disc. Recall that we use the double point with positive sign and recall that $d=2 k$. So we have created spheres $S_{2 i}$ for $i=1, \ldots, k$. For the other spheres, which will form the other half of our set of spherical elements of $H_{2}(W ; \mathbb{Z}[\mathbb{Z}])$, we will use the Whitney discs as below.

Let $p_{1}, p_{2}$ be two double points of $D_{0}$ which have opposite intersection signs and which are paired up by an order one Whitney disc $D_{1}^{i}$. Let $T_{1}$ and $T_{2}$ be the Clifford tori for the double points $p_{1}$ and $p_{2}$ respectively. Let $\alpha$ be the Whitney circle: a curve which goes from 


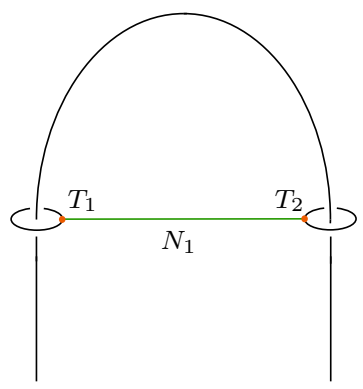

time $= \pm \varepsilon$

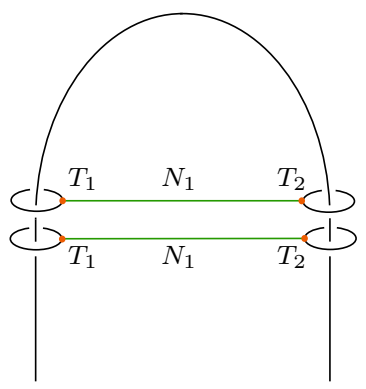

time $= \pm \frac{\varepsilon}{2}$

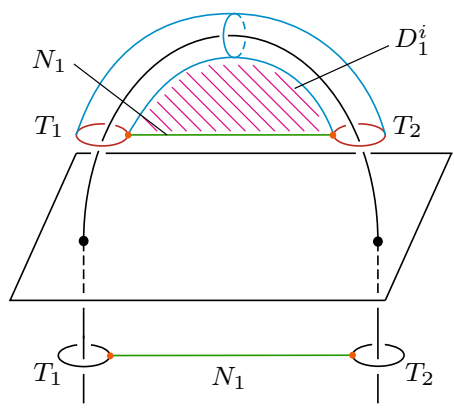

time $=0$

Fig. 4 A picture in $\mathbb{R}^{4} \cong \mathbb{R}^{3} \times \mathbb{R}$ of a model for two intersection points, paired up with a Whitney disc, together with their Clifford tori $T_{1}$ and $T_{2}$ and the annuli $N_{1}$ and $N_{2}$. The last $\mathbb{R}$ coordinate is the time. The future and the past are drawn in the same pictures, to avoid repetition. Note that this is only a model. In reality, since the Whitney disc may not be embedded, all these surfaces may not be contained in one contractible open neighbourhood

$p_{1}$ to $p_{2}$ on $D_{0}$, changes sheets, and then returns to $p_{1}$ on the opposite sheet to the sheet it left on. Write $\alpha=\alpha_{1} \cup \alpha_{2}$, dividing $\alpha$ into two Whitney arcs by cutting at $p_{1}$ and $p_{2}$.

Define two annuli in a similar manner to above. Take the normal circle bundle to $\alpha_{i}$ and consider its intersection with $\partial W$. We obtain $N_{i}:=\left(\alpha_{i} \times S^{1}\right) \cap \partial W$. The boundary of $N_{1}$ is a standard basis curve on $T_{1}$ which we shall call the meridian of $T_{1}$, together with a standard basis curve of $T_{2}$ which we shall call the meridian of $T_{2}$. The boundary of $N_{2}$ are other standard basis curves, which we shall call the longitudes of $T_{1}$ and $T_{2}$. A movie of two Clifford tori, the annuli $N_{1}$ and $N_{2}$, and the Whitney disc $D_{1}^{i}$ is shown in Fig. 4 . In this figure, the past and future pictures are drawn only once, since the situation is symmetric about the zero time slice, time $=0$.

Now we have a three step process. First use two push-offs $N_{1}^{ \pm}$of $N_{1}$ to perform surgery on $T_{1}$ and $T_{2}$ to join them into one bigger torus

$$
T_{12}:=N_{1}^{-} \cup N_{1}^{+} \cup \operatorname{cl}\left(T_{1} \backslash\left(S^{1} \times D^{1}\right)\right) \cup \operatorname{cl}\left(T_{2} \backslash\left(S^{1} \times D^{1}\right)\right) .
$$

Next use two push-offs $\left(D_{1}^{i}\right)_{ \pm}$of the Whitney disc to convert $N_{2}$ into a disc

$$
C:=\operatorname{cl}\left(N_{2} \backslash\left(\alpha_{2} \times D^{1}\right)\right) \cup\left(D_{1}^{i}\right)_{+} \cup\left(D_{1}^{i}\right)_{-} .
$$

Here we abuse notation and also denote the push-off of $\alpha_{2}$ onto $N_{2}$ along $D_{1}^{i}$ by $\alpha_{2}$.

Recall that the boundary of $N_{2}$ was a longitude of $T_{1}$ and a longitude of $T_{2}$. These longitudes have been cut by the surgery which converted $T_{1} \cup T_{2}$ into $T_{12}$. They can be joined by a pair of arcs, $\alpha_{1}^{+}$in $N_{1}^{+}$and $\alpha_{1}^{-}$in $N_{1}^{-}$, to create a longer loop which is a longitude of $T_{12}$, and is also the boundary of $C$. The final step is to use two push-offs of $C$ to perform surgery on $T_{12}$ and create the desired sphere $S_{2 i-1}$. The schematic arrangement of the constituent parts of $S_{2 i-1}$ are shown in Fig. 5.

This completes our description of the spheres $S_{i}$, for $i=1, \ldots, 2 k=d$. Recall that we called the submodule in $\pi_{2}(W)$ they generate $F$. Next we will show that $F$ and $\pi_{2}(W)$ have the same rank, which is equal to the number of double points of $D_{0}$.

Lemma 4.2 Both $H_{2}(W ; \mathbb{Z}[\mathbb{Z}]) \cong \pi_{2}(W)$ and its submodule $F$ are free $\mathbb{Z}[\mathbb{Z}]$-modules of rank $d$.

Together with Lemma 4.1, this proves (1) of Theorems 3.1 and 3.2. 


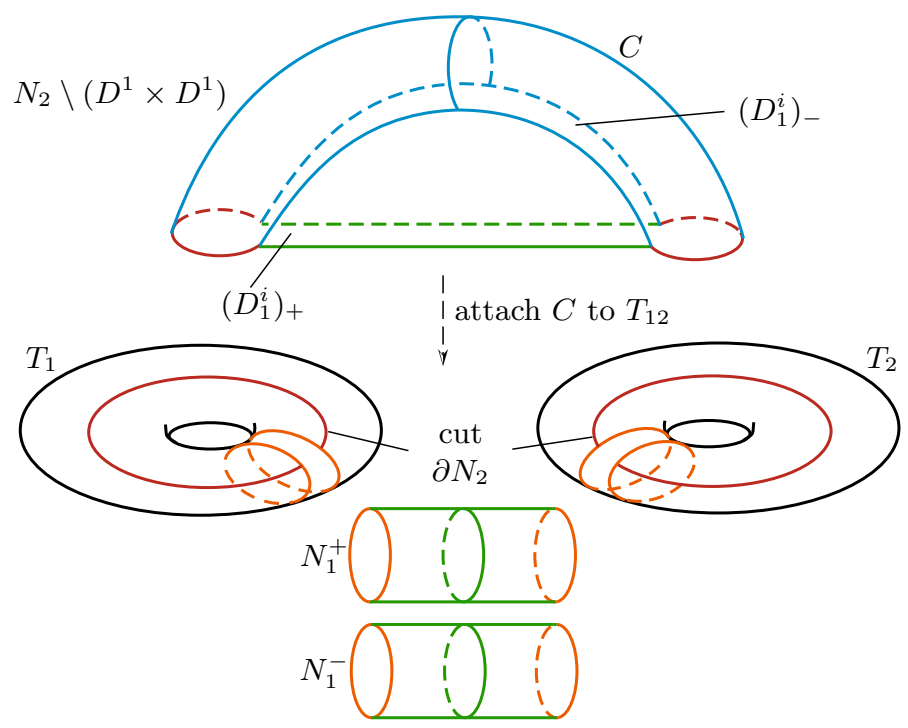

Fig. 5 Schematic diagram of the construction of the disc $C$ from surgery on $N_{2}$ using $D_{1}^{i}$, and the construction of $T_{12}$ from the Clifford tori $T_{1}$ and $T_{2}$ and two parallel copies $N_{1}^{ \pm}$of the annulus $N_{1}$

Proof The fact that $\pi_{1}(W) \cong \mathbb{Z}$ is crucial for this proof. By Lemma $4.1, H_{2}(W ; \mathbb{Z}[\mathbb{Z}])$ is a free module, so is isomorphic to $\mathbb{Z}[\mathbb{Z}]^{\delta}$ for some $\delta$.

Claim $H_{2}(W ; \mathbb{Z}[\mathbb{Z}]) \otimes_{\mathbb{Z}[\mathbb{Z}]} \mathbb{Z} \cong H_{2}(W ; \mathbb{Z})$.

We use the universal coefficient spectral sequence for homology [25, Theorem 5.6.4]

$$
E_{2}^{p, q}=\operatorname{Tor}_{p}^{R}\left(H_{q}(W ; \mathbb{Z}[\mathbb{Z}]), \mathbb{Z}\right) \Longrightarrow H_{n}(W ; \mathbb{Z})
$$

to compute $H_{2}(W ; \mathbb{Z})$ from $H_{*}(W ; \mathbb{Z}[\mathbb{Z}])$. Here the differential $d_{r}$ has degree $(-r, r-1)$. The only nontrivial $E^{2}$ term on the line $p+q=2$ is $E_{2,0}^{2}=H_{2}(W ; \mathbb{Z}[\mathbb{Z}]) \otimes_{\mathbb{Z}[\mathbb{Z}]} \mathbb{Z}$, since $H_{1}(W ; \mathbb{Z}[\mathbb{Z}])=0$ and $H_{0}(W ; \mathbb{Z}[\mathbb{Z}]) \cong \mathbb{Z}$ admits a length one projective $\mathbb{Z}[\mathbb{Z}]$ module resolution $\mathbb{Z}[\mathbb{Z}] \stackrel{t-1}{\longrightarrow} \mathbb{Z}[\mathbb{Z}] \rightarrow \mathbb{Z}$. The differentials $d^{r}$ into $E_{2,0}^{r}(r \geq 2)$ have trivial domains and thus are trivial, since $H_{1}(W ; \mathbb{Z}[\mathbb{Z}]) \cong 0$ and since $\mathbb{Z}[\mathbb{Z}]$ has homological dimension two. This completes the proof of the claim.

Therefore $H_{2}(W ; \mathbb{Z}) \cong \mathbb{Z}[\mathbb{Z}]^{\delta} \otimes_{\mathbb{Z}[\mathbb{Z}]} \mathbb{Z} \cong \mathbb{Z}^{\delta}$. Now we have a second claim:

Claim The second homology is $H_{2}(W ; \mathbb{Z}) \cong \mathbb{Z}^{d}$, generated by the spheres $S_{i}$.

Note that the claim proves (3) of Theorems 3.1 and 3.2. Assuming the claim it follows from $\mathbb{Z}^{\delta} \cong \mathbb{Z}^{d}$ that $\delta=d$. It also follows that the spheres generating $F$ define linearly independent elements of $\pi_{2}(W) \cong H_{2}(W ; \mathbb{Z}[\mathbb{Z}])$. To see this, note that each sphere $S_{i}$ lifts to a nontrivial element of $H_{2}(W ; \mathbb{Z}[\mathbb{Z}])$; let $\mathbb{Z}[\mathbb{Z}]^{d} \rightarrow H_{2}(W ; \mathbb{Z}[\mathbb{Z}]) \cong \mathbb{Z}[\mathbb{Z}]^{d}$ be the homomorphism sending the $i$ th basis to $\left[S_{i}\right]$, and let $P(t)$ be the associated square matrix over $\mathbb{Z}[\mathbb{Z}$. The claim implies that $\operatorname{det} P(1)= \pm 1$. It follows that $\operatorname{det} P(t) \neq 0$, that is, $\mathbb{Z}[\mathbb{Z}]^{d} \rightarrow H_{2}(W ; \mathbb{Z}[\mathbb{Z}])$ is injective. So $F$ has rank $d$.

It remains to prove the claim that $H_{2}(W ; \mathbb{Z}) \cong \mathbb{Z}^{d}$. Recall that $\partial_{+}=\operatorname{cl}\left(\partial\left(v D_{0}\right) \backslash v K\right)$. Let $\partial_{-}=v K$. We have: 


$$
\begin{aligned}
H_{2}(W ; \mathbb{Z}) & \cong H_{3}\left(D^{4}, W ; \mathbb{Z}\right) & & \text { by the long exact sequence for }\left(D^{4}, W\right), \\
& \cong H_{3}\left(v D_{0}, \partial_{+} ; \mathbb{Z}\right) & & \text { by excision, } \\
& \cong H^{1}\left(v D_{0}, \partial_{-} ; \mathbb{Z}\right) & & \text { by duality, } \\
& \cong H^{1}\left(D_{0}, \partial D_{0} ; \mathbb{Z}\right) \cong H_{1}\left(D_{0} ; \mathbb{Z}\right)=\mathbb{Z}^{d}, & & \text { generated by the double point loops. }
\end{aligned}
$$

It follows that the Clifford tori, which are dual to the double point loops, form a basis for $H_{2}(W ; \mathbb{Z})$. The Clifford tori, after a basis change, are homologous to the spheres $S_{i}$, since the $S_{i}$ are obtained from surgery on (linear combinations of) the Clifford tori. This completes the proof of the claim and therefore of Lemma 4.2.

Remark 4.3 In the case of accessory spheres only, the final basis change is not required. Also, note that unfortunately we do not know that $F=\pi_{2}(W)$, only that the two are both free modules of the same rank and that the generators of $F$ give a basis over $\mathbb{Z}$. Therefore, choosing a basis for $\pi_{2}(W)$ and representing the generators of $F$ as vectors, and then making these vectors the columns of a matrix, yields a matrix $P(t)$ which augments to be unimodular. This matrix appeared in the proof of linear independence above and it will appear in the proofs in Sects. 6, 9 and 10 . In the special case that $D_{0}$ arises from crossing changes, we will see in Lemma 6.2 that $F=\pi_{2}(W)$.

\subsection{Definitions of Whitney and accessory framings}

In this section we recall the precise definition of the Whitney framing of the boundary of a Whitney disc. Note that a normal bundle to a surface in 4-dimensional space has 2dimensional fibre. An orientation of the surface and an orientation of the ambient space determines an orientation of the normal bundle. Thus a single nonvanishing vector field in the normal bundle of a surface determines two nonvanishing vector fields, up to homotopy, and therefore a framing. The second vector is chosen so as to be consistent with the orientations.

Definition 4.4 (Whitney framing) Suppose that we have two surfaces, or two sheets of the same surface, $\Sigma_{1}$ and $\Sigma_{2}$, intersecting in two points $p$ and $q$ of opposite signs. Let $\gamma_{i}$ be an arc on $\Sigma_{i}$ between $p$ and $q$, such that $\gamma_{1} \cup \gamma_{2}$ bounds a Whitney disc $D_{1}$. We will describe a framing of $\left.v_{D_{1}}\right|_{\partial D_{1}}$. Choose a framing of $v_{\gamma_{1} \subset \Sigma_{1}}$, a nonvanishing vector field in the normal bundle of $\gamma_{1}$ in $\Sigma_{1}$. This yields a nonvanishing vector field in $\left.v_{D_{1}}\right|_{\gamma_{1}}$. Along $\gamma_{2}$ we choose a vector field in $\left.v_{D_{1}}\right|_{\gamma_{2}} \cap v_{\Sigma_{2}}$, which agrees at $p$ and $q$ with the vector field along $\gamma_{1}$ which we have already chosen (for this to be possible we need that $p$ and $q$ are of opposite signs.) Note that the intersection $\left.v_{D_{1}}\right|_{\gamma_{2}} \cap v_{\Sigma_{2}}$ is a 1-dimensional bundle. The resulting framing along $\partial D_{1}=\gamma_{1} \cup \gamma_{2}$ is the Whitney framing. The transport of the Whitney framing to $\partial W$ along $D_{1}$ is depicted in Fig. 6.

Compare this framing to the disc framing, that is the unique framing of the normal bundle to $D_{1}^{i}$ restricted to $\partial D_{1}^{i}$, in order to obtain the twisting coefficient $a_{i} \in \mathbb{Z}$ of $D_{1}^{i}$. Recall that for the purposes of assigning an integer $a_{i}$, the disc framing is considered to be the zero framing. A Whitney disc is said to be framed if and only if it has coefficient 0 ; equivalently a Whitney disc is framed if the Whitney framing and the disc framing coincide.

As remarked in the introduction, interior twists change the disc framing by \pm 2 relative to the Whitney framing, so we can arrange that the twisting coefficient is either 1 or 0 . Whether or not this step is performed, the entries of $\lambda$ (and therefore of the matrix $\Omega$ ) do not change.

While the Whitney framing defined above is standard (see [22, pages 54-8] for a nice exposition), a framing of the boundary of an accessory disc does not seem to be standard. 


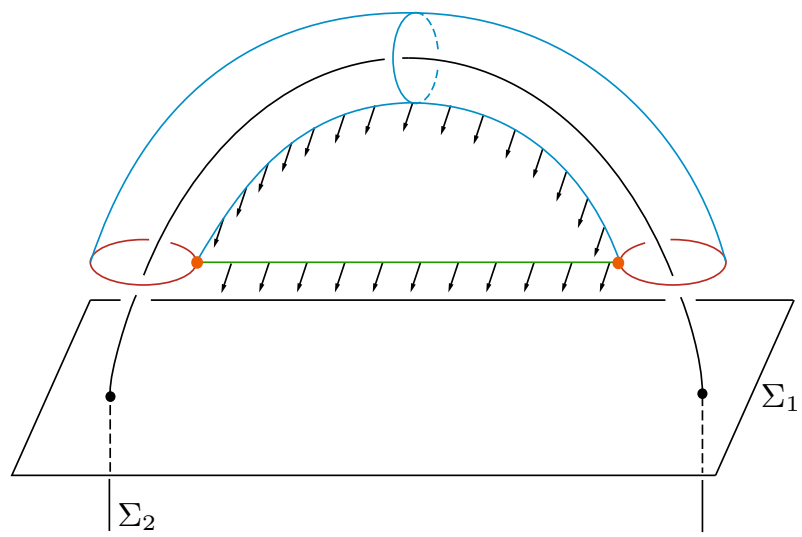

Fig. 6 The Whitney framing of the normal bundle of a Whitney disc along the boundary. It is tangent to $\Sigma_{1}$, which appears in the picture as a plane, but normal to the $\Sigma_{2}$, which is the surface that appears as a line in the picture

Fig. 7 The accessory framing

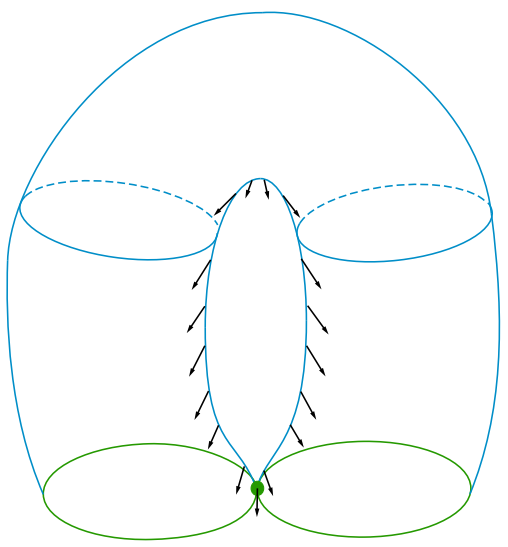

However we will need a detailed understanding of this in order to compute the matrix of the intersection form of $W$.

Definition 4.5 (Accessory framing) Consider the double point loop $\gamma$ of an intersection point $p$ of $D_{0}$, which bounds an accessory disc $A_{1}^{i}$. By restricting the normal bundle of $D_{0}$ to $\gamma$, and looking at $W \cap \partial\left(\left.\operatorname{cl}\left(v D_{0}\right)\right|_{\gamma}\right)$, we obtain the image $N$ of a map into $W$ of an annulus. Define the curve $\gamma^{\prime}:=A_{1}^{i} \cap N$. The boundary $\partial N$ is the union of a longitude and a meridian of the Clifford torus $T$ of the double point $p$. Two points $q_{1}, q_{2}$ on $\partial N$, one on each component of $\partial N \cong S^{1} \times S^{0}$, are identified, where the longitude and meridian of the Clifford torus meet. Thus $\gamma^{\prime}$ is a simple closed curve; in fact $\gamma^{\prime}=\partial A_{1}^{i}$.

Define the accessory framing (or $N$-tangential framing) of $A_{1}^{i}$ restricted to $\gamma^{\prime}=\partial A_{1}^{i}$ to be a framing of the normal bundle of $\gamma^{\prime}$ by a nonzero vector field in the tangent bundle to $N$, except with a slight modification in a neighbourhood of $q=q_{1}=q_{2}$ that moves the vector field away from the tangent bundle $T N$, as shown in Fig. 7; this modification is necessary in order for the framing to be well defined at $q$.

More precisely, near an $\mathbb{R}^{2} \times \mathbb{R}^{2}$ neighbourhood of $q=0$ in which the sheets are $\mathbb{R}^{2} \times 0$ and $0 \times \mathbb{R}^{2}$, we have $T=S^{1} \times S^{1}, N=\left(S^{1} \times \mathbb{R}_{\geq 1} \times 0\right) \cup\left(0 \times \mathbb{R}_{\geq 1} \times S^{1}\right), A_{1}^{i}=0 \times \mathbb{R}_{\geq 1} \times \mathbb{R}_{\geq 1} \times 0$, 
and $\gamma^{\prime}=\left(0 \times 1 \times \mathbb{R}_{\geq 1} \times 0\right) \cup\left(0 \times \mathbb{R}_{\geq 1} \times 1 \times 0\right)$. The framing is $(1,0,0,0)$ on $0 \times 1 \times \mathbb{R}_{\geq 2} \times 0$, is $(0,0,0,1)$ on $0 \times \mathbb{R}_{\geq 2} \times 1 \times 0$, and is of the form $(\cos \theta, 0,0, \sin \theta)$ with $0 \leq \theta \leq \pi / 2$ on the remaining part. Specifically,

$$
(\cos (\pi t / 4), 0,0, \sin (\pi t / 4)) \text { on } \begin{cases}(0,1,2-t, 0) & t \in[0,1] \\ (0, t, 1,0) & t \in[1,2] .\end{cases}
$$

We remark that this is equal to the framing used by Casson [3] for the construction of a Casson handle.

Compare the accessory framing to the disc framing of $A_{1}^{i}$, in order to compute the twisting coefficient $b_{i} \in \mathbb{Z}$ which occurs in the diagonal terms $\lambda_{2 i}$ of $\lambda$. Recall that for the purposes of assigning an integer $b_{i}$, the disc framing is considered to be the zero framing.

\subsection{Equivariant intersections of the spheres $S_{i}$}

Sections 4.4-4.9 describe the intersections amongst the spheres $S_{i}$. Together these sections prove (2), (4) and (5) of Theorem 3.1. Only the computations of Sects. 4.4, 4.7 and 4.9 are required for the proof of (2), (4) and (5) of Theorem 3.2.

We begin with a lemma translating intersections with a Whitney or accessory disc into the intersection numbers from the intersections with a sphere $S_{i}$.

In the next lemma let $\Sigma$ be a surface in $W$ with a path from a basepoint of $\Sigma$ to the basepoint of $W$, for which $\pi_{1}(\Sigma) \rightarrow \pi_{1}(W)$ is the trivial map. Recall that $z:=(1-t)\left(1-t^{-1}\right)$.

Lemma 4.6 For each intersection point of $\Sigma \cap D_{1}^{j}$ (respectively $\Sigma \cap A_{1}^{j}$ ), there are four resulting intersections of $\Sigma \cap S_{2 j-1}$ (respectively $\Sigma \cap S_{2 j}$ ). If the $\mathbb{Z}[\mathbb{Z}]$ intersection number of the intersections of $\Sigma$ with $D_{1}^{j}$ (respectively $A_{1}^{j}$ ) is $p(t)$, then the $\mathbb{Z}[\mathbb{Z}]$ intersection number with $S_{2 j-1}$ (respectively $S_{2 j}$ ) is $z \cdot p(t)$.

Proof We discuss the case of Whitney discs and odd indexed spheres first. Assume that there is a single intersection point in $\Sigma \cap D_{1}^{j}$ and it has $\mathbb{Z}[\mathbb{Z}]$ intersection number +1 .

Consider the four copies of the Whitney disc $D_{1}^{j}$ which occur in $S_{2 j-1}$. First we use two copies of $D_{1}^{j}$ to surger an annulus $N_{2}$ into a disc $C$. These copies of $D_{1}^{j}$ are called $\left(D_{1}^{j}\right)_{ \pm}$. Label so that going from $\left(D_{1}^{j}\right)_{+}$to $\left(D_{1}^{j}\right)_{-}$along $N_{2}$ involves traversing a meridian of $D_{0}$ in the positive sense.

Then we use two copies $C_{ \pm}$of $C$ to surger the torus $T_{12}$. Label so that going from $C_{+}$ to $C_{-}$along $T_{12}$ involves traversing a meridian of $D_{0}$ in the negative sense. Creating $C_{+}$ and $C_{-}$requires two copies of each of $\left(D_{1}^{j}\right)_{ \pm}$, which we call $\left(D_{1}^{j}\right)_{ \pm \pm}$. Observe that $C_{+}$uses $\left(D_{1}^{j}\right)_{++}$and $\left(D_{1}^{j}\right)_{-+}$, while $C_{-}$uses $\left(D_{1}^{j}\right)_{+-}$and $\left(D_{1}^{j}\right)_{--}$. If $\Sigma$ intersects $D_{1}^{j}$ in a point then $\Sigma$ intersects each of the $\left(D_{1}^{j}\right)_{ \pm \pm}$in a point.

In order for $S_{2 j-1}$ to be oriented, we need to take the opposite orientations on $\left(D_{1}^{j}\right)_{+-}$ and $\left(D_{1}^{j}\right)_{-+}$. Choose the orientation of $S_{2 j-1}$ to be such that the intersection signs for $\Sigma \cap\left(D_{1}^{j}\right)_{\zeta \xi}$ is equal to $\zeta \cdot \xi$ for $\zeta, \xi \in\{+,-\}$.

We are given a choice of path from the basepoint of $W$ to the basepoint of $D_{1}^{j}$. Use the same path, perturbed slightly, with the basepoint of $S_{2 i-1}$ located on $D_{++}^{j}$. With respect to this choice of basepoint, the contributions from the intersections of $\Sigma$ with $\left(D_{1}^{j}\right)_{++},\left(D_{1}^{j}\right)_{+-},\left(D_{1}^{j}\right)_{--},\left(D_{1}^{j}\right)_{-+}$are $+1,-t^{-1},+1,-t$ respectively. The sum is $2-t-t^{-1}=(1-t)\left(1-t^{-1}\right)=z$. See Fig. 8 . 
Fig. 8 A schematic diagram of four intersection points of a sphere $S_{2 i-1}$ (or $S_{2 i}$ ) with a surface $\Sigma$ arising from a single intersection point of $D_{i}^{1}\left(\right.$ or $\left.A_{i}^{1}\right)$ with $\Sigma$

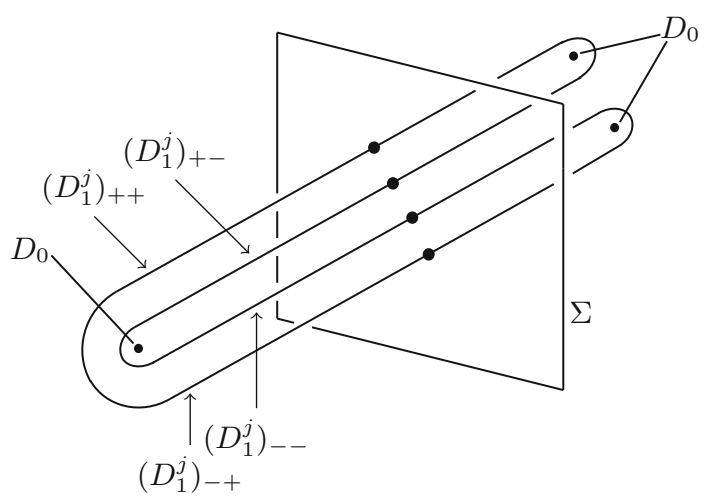

Add the contributions from multiple intersection points in $D_{1}^{j} \cap \Sigma$ to obtain the desired result. If the initial $\mathbb{Z}[\mathbb{Z}]$ intersection number of a point of intersection between $D_{1}^{j}$ and $\Sigma$ is $\pm t^{\ell}$, then the contribution to the intersection number of $\Sigma$ with $S_{2 j-1}$ is $\pm z t^{\ell}$.

The result for the intersection number of the sphere $S_{2 j}$ with a surface $\Sigma$ in terms of the intersection number of $\Sigma$ with $A_{1}^{j}$ is proved in the same way, with $A_{1}^{j}$ replacing $D_{1}^{j}, N$ replacing $N_{2}, D$ replacing $C$, and with $T$ replacing $T_{12}$.

\subsection{Intersection of $S_{i}$ with $S_{j}$ for $i \neq j$ and $\{i, j\} \neq\{2 i-1,2 i\}$}

First we consider the intersections between the spheres $S_{2 i-1}$ and $S_{2 j-1}$ for $i \neq j$. The $\mathbb{Z}[\mathbb{Z}]$ intersections between the spheres $S_{2 i-1}$ and $S_{2 j-1}$ for $i \neq j$ arise directly from intersections between the order one Whitney discs $D_{1}^{i}$ and $D_{1}^{j}$.

We investigate the contribution of a single intersection point between $D_{1}^{i}$ and $D_{1}^{j}$ with associated element $\pm t^{\ell}$. Since $S_{2 i-1}$ contains 4 parallel copies of $D_{1}^{i}$ and $S_{2 j-1}$ contains 4 parallel copies of $D_{1}^{j}$, there are 16 intersection points in $S_{2 i-1} \cap S_{2 j-1}$ arising from the single intersection point in $D_{1}^{i} \cap D_{1}^{j}$.

We apply Lemma 4.6 five times, once with $\Sigma=D_{1}^{i}$ and $D_{1}^{j}$ as the intersecting disc, and then once with $\Sigma$ as each of the four parallel copies of $D_{1}^{j}$ in $S_{2 j-1}$, and $D_{1}^{i}$ the intersecting disc. The resulting $\mathbb{Z}[\mathbb{Z}]$-intersection number is therefore $\pm z^{2} t^{\ell}$.

The intersections of $S_{2 i-1}$ with $S_{2 j}$ for $i \neq j$ and the intersection of $S_{2 i}$ with $S_{2 j}$ for $i \neq j$ are computed in the same way, except that a sphere with even index $S_{2 i}$ contains four parallel copies of an accessory disc instead of a Whitney disc.

\subsection{Intersection of $S_{2 i-1}$ and $S_{2 i}$}

During the construction of $S_{2 i-1}$ and $S_{2 i}$ we must be careful to make sure that the intersections are transverse. There is one Clifford torus associated to one of the double points paired up by $D_{1}^{i}$, say $T_{2}$, a parallel copy of which is also used as the Clifford torus $T$ to surger using $A_{1}^{i}$ in the construction of $S_{2 i}$. We may assume that $T_{2}$ and $T$ are associated to a self-intersection point of $D_{0}$ of positive sign. We use a slightly bigger Clifford torus for $T_{2}$ than for $T$. As a result $T$ is disjoint from $S_{2 i-1}$ but $T_{2}$ intersects $A_{1}^{i}$ in a single point. Apply Lemma 4.6 to 


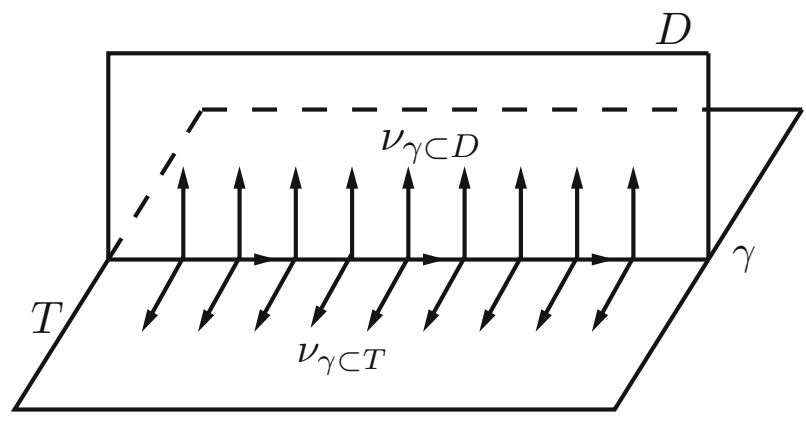

Fig. 9 A 3-dimensional slice of a neighbourhood of a point of $\gamma$, with the surfaces $T$ and $D$ shown, together with trivialisations of the tangent bundle $T \gamma$ and of the normal bundles $v_{\gamma \subset D}$ and $v_{\gamma \subset T}$. The vector field $\mathbf{w}$ is in the 4 th dimension and so is not visible in the picture

obtain a contribution of $z$ to the off-diagonal entries of each $2 \times 2$ block of the matrix $X$ from Theorem 3.1.

\subsection{Framing conditions for surgery}

To understand the self intersection terms, first we need to give a description of the framing conditions that must hold in order for surgery to be performed and the normal bundle of the outcome to again be framed. One can still perform surgery without the framing condition, but then it becomes tricky to verify that one is keeping track of intersection numbers and framing conditions correctly.

Recall that a framing of a surface in a 4-manifold means a framing of its 2-dimensional normal bundle, and a framing is specified by a single nonvanishing vector field in the normal bundle. A second nonvanishing vector field can then be found using the orientation of the normal bundle, which is itself inherited from the orientation of the surface and the orientation of the ambient 4-manifold.

Let $V$ be a 4-manifold, let $T \subset V$ be an embedded torus with trivial normal bundle, with an essential, simple closed curve $\gamma \subset T$, and let $D \rightarrow V$ be an immersed disc which we want to use to perform surgery on $T$, so that $\partial D=\gamma$.

There is a unique framing of $D$ in $W$, that is, trivialisation of the normal bundle $v_{D}$, which we call the disc framing. In addition, suppose we have the following data:

- A framing of $T$ in $W$, which we call a surgery framing.

- A framing $f_{\gamma \subset T}$ of $\gamma \subset T$, that is, a trivialisation of the normal bundle $v_{\gamma \subset T}$.

- A framing $f_{\gamma \subset D}$ of $\gamma \subset D$.

The various vector bundles on $\gamma$ are shown in Fig. 9. Note that the framings of $\gamma \subset T$ and $\gamma \subset D$ are uniquely determined up to negation, while that of $T \subset W$ is not.

In order for the surgery to yield a framed 2-sphere, we require the following: there exists a vector field $\mathbf{w}$ on $\gamma$ such that

(F1) $\left(f_{\gamma \subset T}, \mathbf{w}\right)$ is equivalent to the disc framing on $\gamma$.

(F2) $\left(f_{\gamma \subset D}, \mathbf{w}\right)$ is equivalent to the surgery framing on $\gamma$.

In order for the conditions (F1) and (F2) to hold we might have to make some modifications of the original data. First we may need to boundary twist $D$ around $\gamma$, introducing one intersection in $D \cap T$ for each twist, until there exists a w satisfying (F1). Since it is constrained 
to a single dimension, up to homotopy $\mathbf{w}$ is determined up to sign, and the sign is determined by the other choices of framing. Since $\gamma$ is essential, we are then free to change the surgery framing of $T$ along $\gamma$, until (F2) holds. In the sequel this will always be done without further comment.

We may then use the surgery framing to take two parallel copies of $D$ and construct a framed sphere $S$. The framing on $S$ is obtained by taking the framing on $v D$ on one copy of $D$, its negative on the other copy of $D$, the framing of $\nu T$ on $T \backslash\left(\gamma \times D^{1}\right)$, and then smoothing the corners by rotating between the two vector fields in a neighbourhood of $\gamma \times\{ \pm 1\}$. The rotation occurs in the 2-dimensional subbundle of $\left.T V\right|_{\gamma}$ which is orthogonal to $T \gamma$ and $\mathbf{w}$.

\subsection{Self intersection of $S_{2 i-1}$}

First, we note that each self-intersection of the disc $D_{1}^{i}$ gives rise to 16 self intersection points of $S_{2 i-1}$, which means that we should count 32 intersection points between $S_{2 i-1}$ and a push-off.

Given a self-intersection point $p$ of $D_{1}^{i}$ with double point loop $t^{\ell}$ and sign \pm , the intersection number between $D_{1}^{i}$ and a parallel push-off is $\pm\left(t^{\ell}+t^{-\ell}\right)$. We can only define the double point loop up to the indeterminacy $t^{\ell}=t^{-\ell}$, since we have no canonical ordering of sheets. Of course $t^{\ell}+t^{-\ell}$ is independent of the choice here. Now apply the argument of Sect. 4.5 to yield a coefficient of $z^{2}$, noting that $z=\bar{z}$. This accounts for the diagonal terms of $z\left(z Y+\overline{z Y}^{T}\right)$. There are indeed 32 terms for each $\pm t^{\ell}$ summand of $Y$.

The potential twisting of the Whitney discs gives the crucial extra terms. We want the sphere $S_{2 i-1}$ to be framed, in order to be able to compute the self intersection number $\lambda\left(S_{2 i-1}, S_{2 i-1}\right)$ by counting intersection points between $S_{2 i-1}$ and a parallel push-off. The twisting occurs in the first step, during the construction of $C$ from $N_{2}$ and $\left(D_{1}^{i}\right)_{ \pm}$.

Recall that we denote $\alpha_{1}=N_{1} \cap \partial D_{1}^{i}$ and $\alpha_{2}=N_{2} \cap \partial D_{1}^{i}$. The notation $\alpha_{1}, \alpha_{2}$ was also used for the Whitney arcs which lie on $D_{0}$, so we make a slight abuse to use the same notation for their push-offs onto $N_{1}, N_{2}$ respectively.

Align the disc framing of $D_{1}^{i}$ with the Whitney framing along $\alpha_{1}$. Note that, within the homotopy class, we are free to adjust any framing on an interval. Then look at the disc framing of $D_{1}^{i}$ restricted to $\alpha_{2}$. The difference between this framing and Whitney framing, which is also the surgery framing along $N_{2}$, is the twisting coefficient $a_{i}$. Introduce $a_{i}$ boundary twists along $\alpha_{2}$. Twisting is described in [9, Section 1.3]. (With respect to the whole of the Whitney disc, as originally pairing intersections of $D_{0}$, this is an interior twist. However with respect to the sub-disc whose boundary is $\left(N_{1} \cap \partial D_{1}^{i}\right) \cup\left(N_{2} \cap \partial D_{1}^{i}\right)$, this is a boundary twist. Only the part of the Whitney disc that we use for surgery is relevant.) The boundary twist changes the Whitney disc, and therefore the disc framing, so that it now coincides with the surgery framing along $N_{2}$. Strictly speaking, for these boundary twists, we should push $N_{2}$ slightly off $\partial W$.

The Whitney framing along $N_{1}$ differs from the surgery framing on $T_{12}$ by a fixed rotation. Both are normal to $D_{1}^{i}$ along $N_{1} \cap \partial D_{1}^{i}$. Therefore in a neighbourhood of $\alpha_{1}$ we can arrange the disc framing by a homotopy so that it lies in $v_{\alpha_{1} \subset N_{1}}$.

The disc framing of $C$ is constructed from the disc framing of $\left(D_{1}^{i}\right)_{+}$, the negative of the disc framing of $\left(D_{1}^{i}\right)_{-}$and the normal framing to $N_{2}$. This latter is also the disc framing of $N_{2} \backslash\left(\alpha_{2} \times D^{1}\right)$. The fact that we obtain the disc framing of $C$ is guaranteed by the boundary twists above. For the second surgery, converting $T_{12}$ to $S_{2 i-1}$ using $C_{ \pm}$, the framings already coincide as required by Sect. 4.7. Therefore no more boundary twisting is required. 
Now we consider the contribution of a boundary twist as above to the self intersection number. Each boundary twist produces a single intersection point between $N_{2}$ and $D_{1}^{i}$. It therefore produces two self-intersection points of $C$.

Two copies of $N_{2}$ will be in the final sphere $S_{2 i-1}$. To compute the self intersection number $\lambda\left(S_{2 i-1}, S_{2 i-1}\right)$, first we compute the Wall self intersection $\mu\left(S_{2 i-1}\right)$ [24, Chapter 5], and observe that $\lambda\left(S_{2 i-1}, S_{2 i-1}\right)=\mu\left(S_{2 i-1}\right)+\overline{\mu\left(S_{2 i-1}\right)}$. This works for two reasons. First, the sphere $S_{2 i-1}$ is framed, as we just went to great lengths above to ensure. Thus there is no extra term from the Euler characteristic of the normal bundle [24, Theorem 5.2 (iii)]. Second, although the self-intersection $\mu\left(S_{2 i-1}\right)$ is only well-defined up to the indeterminacy $a=\bar{a}$, the sum $\mu\left(S_{2 i-1}\right)+\overline{\mu\left(S_{2 i-1}\right)}$ is well-defined and determines a unique element of $\mathbb{Z}[\mathbb{Z}]$.

Label the two copies of $N_{2}$ which occur in $C_{ \pm}$by $\left(N_{2}\right)_{ \pm}$. The intersection numbers of these with $D_{1}^{j}$ are 1 and $-t$ respectively, since the two intersections differ by a meridian of $D_{0}$. By Lemma 4.6, the contribution to the self intersection number from each boundary twist is therefore $(1-t) z$. Therefore the contribution to $\lambda\left(S_{2 i-1}, S_{2 i-1}\right)$ is

$$
(1-t) z+\overline{(1-t) z}=\left(1-t+1-t^{-1}\right) z=z^{2} .
$$

All together the boundary twists therefore contribute $a_{i} z^{2}$ to $\lambda\left(S_{2 i-1}, S_{2 i-1}\right)$.

\subsection{Self intersection of $S_{2 i}$}

There are three types of contributions to the self intersection of $S_{2 i}$. First, a self-intersection of the disc $A_{1}^{i}$ with $\mathbb{Z}[\mathbb{Z}]$-intersection number $p(t)$ contributes $z(z p(t)+\overline{z p(t)})$, by the analogous argument as for the spheres $S_{2 i-1}$ in Sect. 4.8.

The twisting $b_{i}$ of the accessory framing (Fig. 7) with respect to the disc framing contributes $b_{i} z^{2}$, by a similar argument to that in Sect. 4.8. We give the outline. Again we need that the disc framing of $D$ is constructed from the disc framings of $N \backslash\left(D^{1} \times D^{1}\right)$ and $A_{+}$ together with the negative of the disc framing of $A_{-}$. To achieve this perform $b_{i}$ boundary twists of $A_{1}^{i}$ around $A_{1}^{i} \cap N$. These contribute $b_{i} z^{2}$ to $\lambda\left(S_{2 i}, S_{2 i}\right)$ as claimed.

In the construction of the spheres $S_{2 i-1}$, the first set of boundary twists was sufficient: after this the second surgery, of $T_{12}$ into a sphere, was automatically correctly framed. However, for the spheres $S_{2 i}$ constructed from the accessory discs, that we consider in this section, this is not the case.

Claim The surgery framing of the $(1,1)$ curve on the Clifford torus $T$ is +1 with respect to the disc framing on $D$.

Given the claim, we perform a single boundary twist of $D$ about its boundary, before using it to surgery $T$ into $S_{2 i}$. This gives rise to a contribution of $1-t$ to the self intersection $\mu\left(S_{2 i}\right)$, therefore a contribution of $\mu\left(S_{2 i}\right)+\overline{\mu\left(S_{2 i}\right)}=1-t+1-t^{-1}=z$ to $\lambda\left(S_{2 i}, S_{2 i}\right)$ as desired.

Roughly, the +1 from the claim arises from the self linking of the $(1,1)$ curve on the Clifford torus. This was previously observed in a different context in [8, Lemma 4]. Note that if the sign of the associated double point of $D_{0}$ were -1 , then the difference in framings would instead be -1 .

The claim will follow from the observation of the next lemma. In order to state the lemma, we describe a disc $D^{\prime}$ in a $D^{4}$ neighbourhood of a double point $p$ of $D_{0}$, whose boundary is the $(1,1)$-curve on the Clifford torus $T$ i.e. the boundaries of $D$ and $D^{\prime}$ coincide. Recall that the Clifford torus is $T=S^{1} \times S^{1} \subset \mathbb{R}^{2} \times \mathbb{R}^{2} \cong \mathbb{R}^{4} \cong D^{4}$. The meridian is $S^{1} \times\{1\}$ and the 
Fig. 10 The framing of the normal bundle of $D^{\prime}$ restricted to $\partial D^{\prime}$, in a neighbourhood of the intersection on $T$ of the meridian and longitude. The shaded triangles are part of $D^{\prime}$, and the fact that the framing stays normal to them means that it twists out of the tangent bundle of $T$
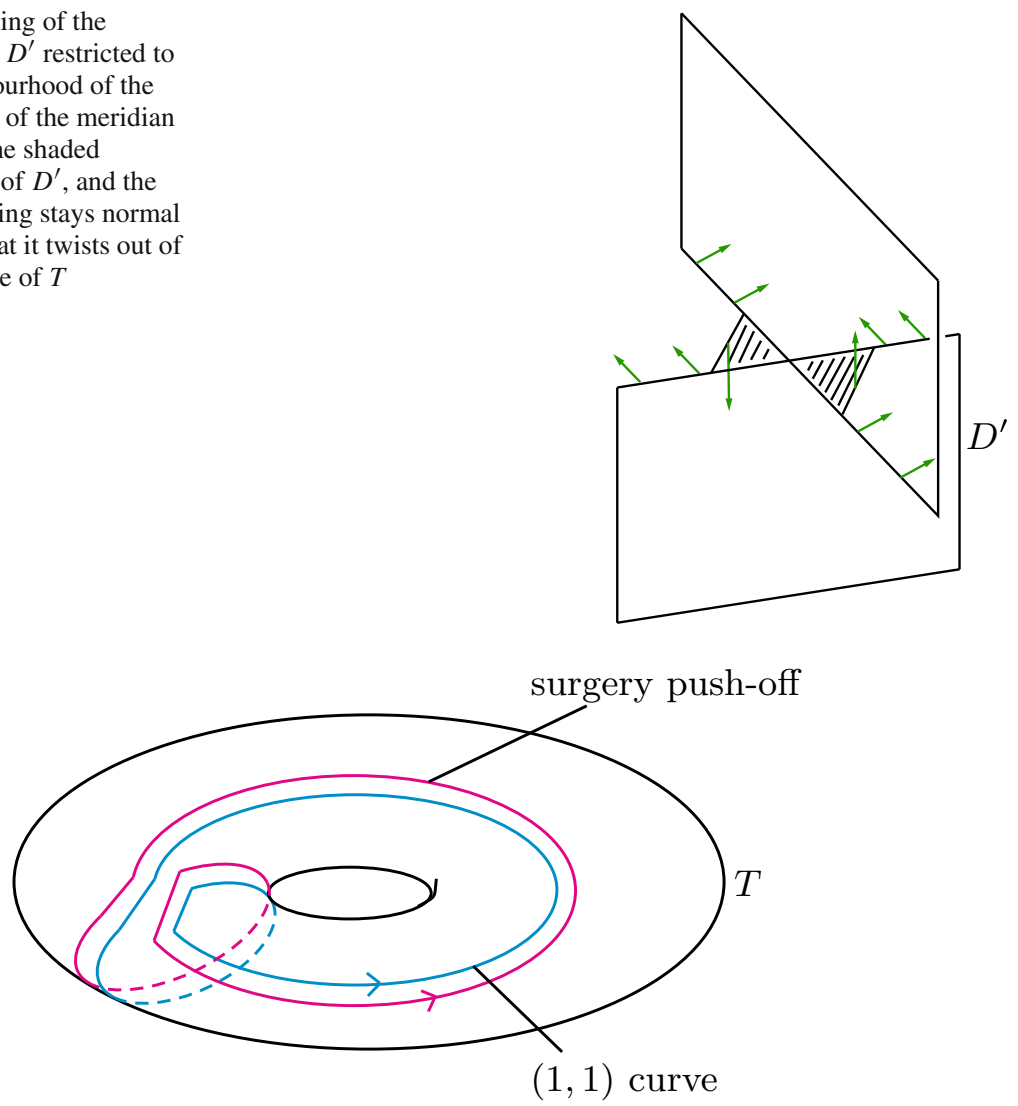

Fig. 11 The $(1,1)$-curve on the Clifford torus $T$ and a push-off using the surgery framing. The linking number in $S^{3}$ is +1

longitude is $\{1\} \times S^{1}$. Take the union of the two discs $D^{2} \times\{1\}$ and $\{1\} \times D^{2}$ and add two small triangles as shown in Fig. 10.

Lemma 4.7 There exists a 3-ball $B$ in $D^{4}$ whose boundary is the 2-sphere formed from the union of the surgery disc $D$ with the disc $D^{\prime}$. Moreover there exists a framing for the normal bundle of $B$ which restricts to the disc framings of both $D$ and $D^{\prime}$.

Proof The 3-ball $B$ is constructed from glueing together $A_{1}^{i} \times[-1,1]$ and $\alpha \times D^{2}$-recall that $A_{1}^{i} \times\{ \pm 1\} \cong A_{ \pm}$and $\alpha \times S^{1}=N$. The normal bundle of $B$ is one dimensional, so the framing only depends on a choice of sign. The framing determines a nonvanishing vector field in the normal bundle of $D$ and $D^{\prime}$, which therefore must restrict to the disc framings on their common normal boundary.

By Lemma 4.7, we can compute the disc framing of $D$ restricted to its boundary by computing the disc framing of $D^{\prime}$. The surgery framing is +1 with respect to the disc framing of $D^{\prime}$. The surgery framing is shown in Fig. 11, where we see that the linking number of the two curves is +1 .

To compute the framing of $D^{\prime}$, isotope it in a collar neighbourhood of the boundary so that a (smaller) collar neighbourhood lies in $S^{3}$. The framing of $D^{\prime}$ and the surgery framing agree 
along the meridian of $T$, are opposite along the longitude, and in a neighbourhood of the intersection point of the longitude and the meridian of $T$ there is a rotation. The arrangement is as shown in Fig. 10. As the framing vector for $D^{\prime}$ stays normal to the two small triangles we see that it undertakes a single full -1 twist with respect to the surgery framing. We compute that the framing of $D^{\prime}$ induces a push-off which has linking number zero with the $(1,1)$ curve of $T$. Thus the surgery framing is +1 with respect to the disc framing. This completes the proof of the claim and therefore of the computation of the self-intersection of the spheres $S_{2 i}$.

\section{Homology of the boundary of $W$}

Proposition 5.1 The first homology $H_{1}(\partial W ; \mathbb{Z}[\mathbb{Z}])$ is isomorphic to $H_{1}\left(X_{K} ; \mathbb{Z}[\mathbb{Z}]\right) \oplus$ $(\mathbb{Z}[\mathbb{Z}] /\langle z\rangle)^{d}$. Consequently the order of $H_{1}(\partial W ; \mathbb{Z}[\mathbb{Z}])$ is $(t-1)^{2 d} \Delta_{K}(t)$.

Proof As before, let $v D_{0}$ be a (closed) regular neighbourhood of the order zero disc $D_{0}$ in $D^{4}$. Since $D_{0}$ has $d$ double points, $v D_{0}$ is obtained by $d$ self plumbings performed on a 2-handle $D^{2} \times D^{2}$. We have $W=\operatorname{cl}\left(D^{4} \backslash v D_{0}\right)$. Let $\partial_{+}=\partial\left(v D_{0}\right) \cap W$ and $\partial_{-}=\operatorname{cl}\left(\partial\left(v D_{0}\right) \backslash \partial_{+}\right)$. Let $X_{K}=\operatorname{cl}\left(S^{3} \backslash \partial_{-}\right)$be the exterior of the knot $K$. Then $\partial W=\partial_{+} \cup X_{K}$ and $\partial_{+} \cap X_{K}=\partial X_{K}$.

The left hand side of Fig. 12 is a surgery description of $\partial\left(v D_{0}\right)=\partial_{+} \cup \partial_{-}$obtained from a standard Kirby diagram of the plumbed handle. More precisely, by choosing double point loops for self plumbings, a homeomorphism between $\partial\left(v D_{0}\right)$ and the 3-manifold given by the surgery description is determined. For the purpose of this section, temporarily choose double point loops whose push-offs along the accessory framing are trivial in $\pi_{1}(W)=\mathbb{Z}$. This can be done by wrapping part of a double point loop on a sheet near the double point, around another sheet, if necessary. (The double point loops used here may be different from those in other sections of the article.) Now, remove the solid torus $\partial_{-}$and take the infinite cyclic cover of $\partial_{+}$. Note that the meridians of the zero-framed circles correspond to push-offs of double point loops along the accessory framing, and so they are trivial in $\pi_{1}(W)=\mathbb{Z}$. It follows that the infinite cyclic cover is given by the surgery diagram in the right hand side of

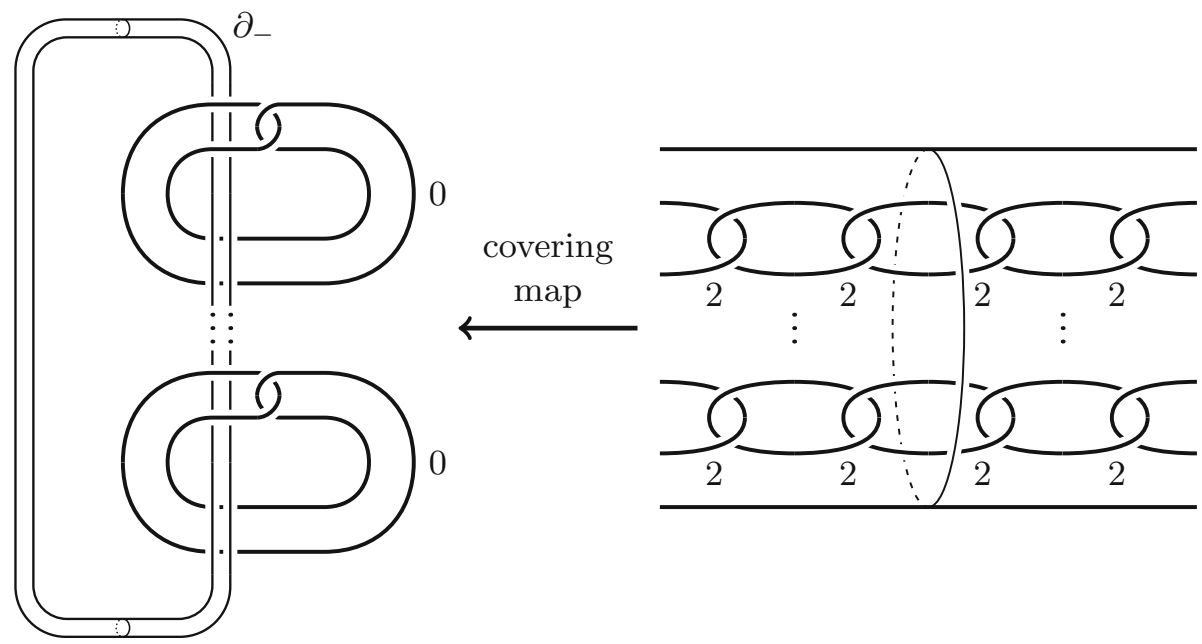

Fig. 12 The 3-manifold $\partial_{+} H$ and its infinite cyclic cover 
Fig. 12, which consists of $d$ infinite chains lying in $D^{2} \times \mathbb{R}$. Observe that the zero framing of the surgery curve in the base corresponds to the \pm 2 framing of the surgery curve in the cover. The signs of the surgery coefficients in the cover and the signs of the clasps are determined by the sign of the double points.

From the surgery description of the infinite cyclic cover, we obtain a presentation of $H_{1}\left(\partial_{+} ; \mathbb{Z}[\mathbb{Z}]\right)$ with $d$ generators, say $v_{i}$, and $d$ defining relations $\pm\left(2-t^{-1}-t\right) v_{i}=0$. It follows that $H_{1}\left(\partial_{+} ; \mathbb{Z}[\mathbb{Z}]\right)=\bigoplus^{d} \mathbb{Z}[\mathbb{Z}] /\left(2-t^{-1}-t\right)$.

Also, $H_{1}\left(\partial X_{K} ; \mathbb{Z}[\mathbb{Z}]\right) \cong \mathbb{Z}$ is generated by a longitude of $K$, which is zero in each of $H_{1}\left(\partial_{+} ; \mathbb{Z}[\mathbb{Z}]\right)$ and $H_{1}\left(X_{K} ; \mathbb{Z}[\mathbb{Z}]\right)$. Therefore, by a Mayer-Vietoris argument for $\partial W=$ $\partial_{+} \cup X_{K}$, we obtain the following, from which the promised conclusion follows immediately.

$$
\begin{aligned}
H_{1}(\partial W ; \mathbb{Z}[\mathbb{Z}]) & \cong H_{1}\left(X_{K} ; \mathbb{Z}[\mathbb{Z}]\right) \oplus H_{1}\left(\partial_{+} ; \mathbb{Z}[\mathbb{Z}]\right) \\
& \cong H_{1}\left(X_{K} ; \mathbb{Z}[\mathbb{Z}]\right) \oplus\left(\mathbb{Z}[\mathbb{Z}] /\left(2-t^{-1}-t\right)\right)^{d} .
\end{aligned}
$$

\section{Proof of Alexander polynomial assertions of main theorems}

We begin with a straightforward lemma.

Lemma 6.1 The relative homology $H_{2}(W, \partial W ; \mathbb{Z}[\mathbb{Z}])$ is isomorphic to $\mathbb{Z}[\mathbb{Z}]^{d}$.

Proof We have isomorphisms

$$
H_{2}(W, \partial W ; \mathbb{Z}[\mathbb{Z}]) \cong H^{2}(W ; \mathbb{Z}[\mathbb{Z}]) \cong \operatorname{Hom}_{\mathbb{Z}[\mathbb{Z}]}\left(H_{2}(W ; \mathbb{Z}[\mathbb{Z}]), \mathbb{Z}[\mathbb{Z}]\right) \cong \mathbb{Z}[\mathbb{Z}]^{d} .
$$

The last isomorphism uses that $H_{2}(W ; \mathbb{Z}[\mathbb{Z}]) \cong \mathbb{Z}[\mathbb{Z}]^{d}$. The second isomorphism uses the universal coefficient spectral sequence

$$
E_{p, q}^{2}=\operatorname{Ext}_{p}^{R}\left(H_{q}(W ; \mathbb{Z}[\mathbb{Z}]), \mathbb{Z}[\mathbb{Z}]\right) \Longrightarrow H^{n}(W ; \mathbb{Z}[\mathbb{Z}])
$$

as we shall now explain. Since $\left.H_{1}(W ; \mathbb{Z}[\mathbb{Z}]), \mathbb{Z}[\mathbb{Z}]\right)=0$ and $\mathbb{Z}$ has a length one projective resolution over $\mathbb{Z}[\mathbb{Z}]$ (see the proof of Lemma 4.2), the only surviving $E_{2}$ term on the line $p+q=2$ is $E_{2}^{0,2}=\operatorname{Hom}_{\mathbb{Z}[\mathbb{Z}]}\left(H_{2}(W ; \mathbb{Z}[\mathbb{Z}]), \mathbb{Z}[\mathbb{Z}]\right)$. The differentials $d_{r}(r \geq 2)$ defined on $E_{r}^{0,2}$ are trivial since $H_{1}(W ; \mathbb{Z}[\mathbb{Z}])=0$ and $\mathbb{Z}[\mathbb{Z}]$ has homological dimension two. Therefore the spectral sequence collapses and we have the isomorphism claimed.

We are ready to connect the pieces of the previous two sections to prove the Alexander polynomial parts of the main theorems. The assertions relating to the Blanchfield form are addressed later in Sect. 10. Theorem 1.4 also uses Lemma 6.2 below.

Proof of Alexander polynomial assertions of Theorems 1.2, 1.3 and 1.4 Since $H_{1}(W ; \mathbb{Z}[\mathbb{Z}]) \cong$ 0 and $H_{2}(W ; \mathbb{Z}[\mathbb{Z}]) \cong H_{2}(W, \partial W ; \mathbb{Z}[\mathbb{Z}]) \cong \mathbb{Z}[\mathbb{Z}]^{d}$, the long exact sequence of a pair yields

$$
\mathbb{Z}[\mathbb{Z}]^{d} \stackrel{\Lambda}{\rightarrow} \mathbb{Z}[\mathbb{Z}]^{d} \longrightarrow H_{1}(\partial W ; \mathbb{Z}[\mathbb{Z}]) \longrightarrow 0
$$

where $\Lambda$ is the intersection form of $W$. Since $H_{1}(\partial W ; \mathbb{Z}[\mathbb{Z}])$ is a torsion module it follows that $\Lambda$ is injective. Indeed

$$
\begin{aligned}
H_{2}(\partial W ; \mathbb{Z}[\mathbb{Z}]) \cong H^{1}(\partial W ; \mathbb{Z}[\mathbb{Z}]) & \cong \operatorname{Ext}_{\mathbb{Z}[\mathbb{Z}]}^{1}\left(H_{0}(\partial W ; \mathbb{Z}[\mathbb{Z}]), \mathbb{Z}[\mathbb{Z}]\right) \\
& \cong \operatorname{Ext}_{\mathbb{Z}[\mathbb{Z}]}^{1}(\mathbb{Z}, \mathbb{Z}[\mathbb{Z}]) \cong \mathbb{Z}
\end{aligned}
$$


and any $\mathbb{Z}[\mathbb{Z}]$-module homomorphism from $\mathbb{Z}$ into a free $\mathbb{Z}[\mathbb{Z}]$ module is trivial.

Represent $\lambda$ as a matrix with respect to the basis for $F \subseteq H_{2}(W ; \mathbb{Z}[\mathbb{Z}])$ defined in Sect. 3, and with respect to a dual basis for $F^{*} \supseteq \operatorname{Hom}_{\mathbb{Z}[\mathbb{Z}]}\left(H_{2}(W ; \mathbb{Z}[\mathbb{Z}]), \mathbb{Z}[\mathbb{Z}]\right) \cong$ $H_{2}(W, \partial W ; \mathbb{Z}[\mathbb{Z}])$, so that we obtain a matrix for the intersection form of $W$ restricted to $F$. The presentation for $H_{1}(\partial W ; \mathbb{Z}[\mathbb{Z}])$ implies that

$$
\operatorname{det}(\Lambda)=\operatorname{ord}_{\mathbb{Z}[\mathbb{Z}]}\left(H_{1}(\partial W ; \mathbb{Z}[\mathbb{Z}])\right) \doteq(t-1)^{2 d} \Delta_{K}(t)
$$

up to multiplication by a unit $\pm t^{m}$. Here we used Proposition 5.1. Up to multiplication by a unit we have $(t-1)^{2} \doteq(1-t)\left(1-t^{-1}\right)=z$. For Theorem 1.2, the matrix $\Omega$ recording intersection data of the Whitney tower satisfies $\lambda=z \Omega$, with $\lambda$ as in Theorem 3.1. Therefore, since $\lambda$ is a $d \times d$ matrix, we have

$$
\operatorname{det}(\lambda)=\operatorname{det}(z \Omega)=z^{d} \operatorname{det} \Omega=(t-1)^{2 d} \operatorname{det}(\Omega)
$$

up to a unit in $\mathbb{Z}[\mathbb{Z}]$. Similarly, with $\lambda$ as in Theorem 3.2, we have

$$
\operatorname{det}(\lambda)=\operatorname{det}(z \Psi)=z^{d} \operatorname{det} \Psi=(t-1)^{2 d} \operatorname{det}(\Psi)
$$

up to a unit in $\mathbb{Z}[\mathbb{Z}]$.

Now suppose that $F=\pi_{2}(W)$. Then $\Lambda=\lambda$ so $(t-1)^{2 d} \Delta_{K}(t)=(t-1)^{2 d} \operatorname{det}(\Psi)$, and cancelling the $(t-1)$ factors yields $\operatorname{det}(\Omega)=\Delta_{K}(t)$. Thus Alexander polynomial assertion of Theorem 1.4 follows from Lemma 6.2 below.

In general, we have that $F \subseteq H_{2}(W ; \mathbb{Z}[\mathbb{Z}])$ is a free module of the same rank. We have a commutative diagram:

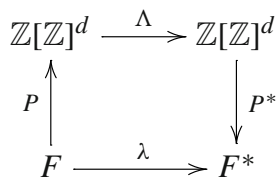

where $P=P(t)$ is represented by a matrix which satisfies $\operatorname{det}(P(1))= \pm 1$.

Then we have

$$
\begin{aligned}
(t-1)^{2 d} \operatorname{det}(\Omega) & =\operatorname{det}(\lambda)=\operatorname{det}(P(t)) \operatorname{det}(\Lambda) \operatorname{det}\left(P(t)^{*}\right) \\
& =\operatorname{det}(P(t)) \operatorname{det}\left(P\left(t^{-1}\right)\right) \operatorname{det}(\Lambda)=f(t) f\left(t^{-1}\right)(t-1)^{2 d} \Delta_{K}(t),
\end{aligned}
$$

where $f(t):=\operatorname{det}(P(t))$. From this we deduce that, modulo norms $f(t) f\left(t^{-1}\right)$ with $f(1)=$ \pm 1 , we have $\operatorname{det}(\Omega)=\Delta_{K}(t)$ as claimed. For Theorem 1.3, replace $\Omega$ with $\Psi$ in the above argument. As remarked above, Theorem 1.4 uses Lemma 6.2 below.

The next lemma completes the proof of the Alexander polynomial assertions of Theorem 1.4 , by showing that in a special case our spheres $S_{i}$, which generate $F$, in fact give a basis for $\pi_{2}(W)$.

Let $D_{0} \leftrightarrow D^{4}$ be an immersed disc in the 4-ball with boundary a knot $K \subset S^{3}$, where $D_{0}$ is produced as the track of a homotopy between $K$ and the unknot, followed by a disc bounding the unknot, where all self-intersection points of the homotopy occur at time $1 / 2$, corresponding to $d$ crossing changes of the knot. More precisely, let $f: S^{1} \times I \rightarrow S^{3}$ be a homotopy with $f\left(S^{1},\{s\}\right)$ isotopic to $K$ for $s<1 / 2$, isotopic to $U$ for $s>1 / 2$, and $f\left(S^{1},\{1 / 2\}\right)$ a singular knot with $d$ double points. The track of the homotopy is the image of $g: S^{1} \times I \rightarrow S^{3} \times I$ given by $g(x, s)=(f(x, s), s)$. Cap off $S^{3} \times\{1\}$ with a copy of $D^{4}$ and cap off $U \subset S^{3} \times\{1\}$ with a standard slice disc for the unknot in this $D^{4}$. 
Fig. 13 A 2-handle which we add at each marked crossing of the unknotted dotted circle. Altering these crossings produces $K$. This constructs a handle decomposition of the exterior of the immersed disc determined by these crossing changes. The two straight strands represent part of the unknotted dotted circle

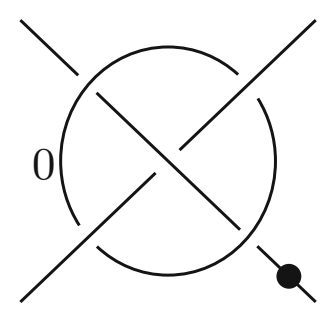

The Clifford tori for the double points can be surgered into 2-spheres $S_{i}$, where $i=$ $1, \ldots, d$, using accessory discs, just as in the construction of the spheres $S_{2 i}$ in Sect. 4.2. As usual define $W:=D^{4} \backslash v D_{0}$.

Lemma 6.2 The 2-spheres $S_{i}$ form a basis for $\pi_{2}(W)$.

Proof We construct a handle decomposition for $W$. Start with a 0-handle and a single 1handle. Represent this by a Kirby diagram with a single dotted unknot. Perform an isotopy of this unknot until it is represented by a diagram having a set of marked crossings (potentially a proper subset of all the crossings) which, if changed, yield the knot $K$. At each such crossing, add a single 0-framed 2-handle in the configuration shown in Fig. 13.

Detailed justification for this can be found in [16, Proposition 3.1], which we now summarise. The exterior of an immersed disc constructed by a crossing change on a knot can be understood in two steps as follows, which occur at the level sets $1 / 2 \pm \varepsilon$ of the function $F$ given by projection to the $I$ factor of $S^{3} \times I$, restricted to the exterior of $D_{0}$. Since we are now passing from $U$ to $K$, we move in the direction of decreasing $I$ factor. First, at $1 / 2+\varepsilon$, remove a small vertical arc which connects the two strands of the crossing. One observes that removing the neighbourhood of an arc as described does not change the diffeomorphism type i.e. the diffeomorphism type of $F^{-1}([a, 1])$ does not change when $a$ crosses $1 / 2+\varepsilon$. The crossing may be switched by sliding the arcs of the knot (the dotted circle) up and down along the removed arc. Then replace the neighbourhood of the vertical arc. Replacing the arc is equivalent to adding the 2-handle as shown in Fig. 13, since this figure shows the crossing of the unknotted circle, that is before the sliding of the arcs (once the crossing is changed, the 2-handle attaching circle bounds a disc in between crossing strands).

Note that $\pi_{1}(W) \cong \mathbb{Z}$, since there is a unique 1-handle and all 2-handles have no effect on the fundamental group. A chain complex $C_{*}(W ; \mathbb{Z}[\mathbb{Z}])$ is given by (compare [16, Proposition 4.4])

$$
\mathbb{Z}[\mathbb{Z}]^{d}=C_{2} \stackrel{0}{\rightarrow} \mathbb{Z}[\mathbb{Z}]=C_{1} \stackrel{t-1}{\longrightarrow} \mathbb{Z}[\mathbb{Z}]=C_{0} .
$$

From this we compute $H_{2}(W ; \mathbb{Z}[\mathbb{Z}]) \cong \mathbb{Z}[\mathbb{Z}]^{d}$ and we note that the set of 2-handles give a basis. The Clifford torus can be seen as the core of each 2-handle, union the punctured torus constructed by taking a disc bounded by the zero-framed component in Fig. 13, which intersects the knot in two points, and tubing along the knot. The double point loop (after suitable twisting) is null homotopic in the complement of the standard slice disc for the unknot found in time $s>1 / 2$, therefore the Clifford torus can be surgered to a sphere using the procedure of Sect. 4.2. Since the core of the 2-handle is still used precisely once, this therefore represents a basis element of $\pi_{2}(W)$. 

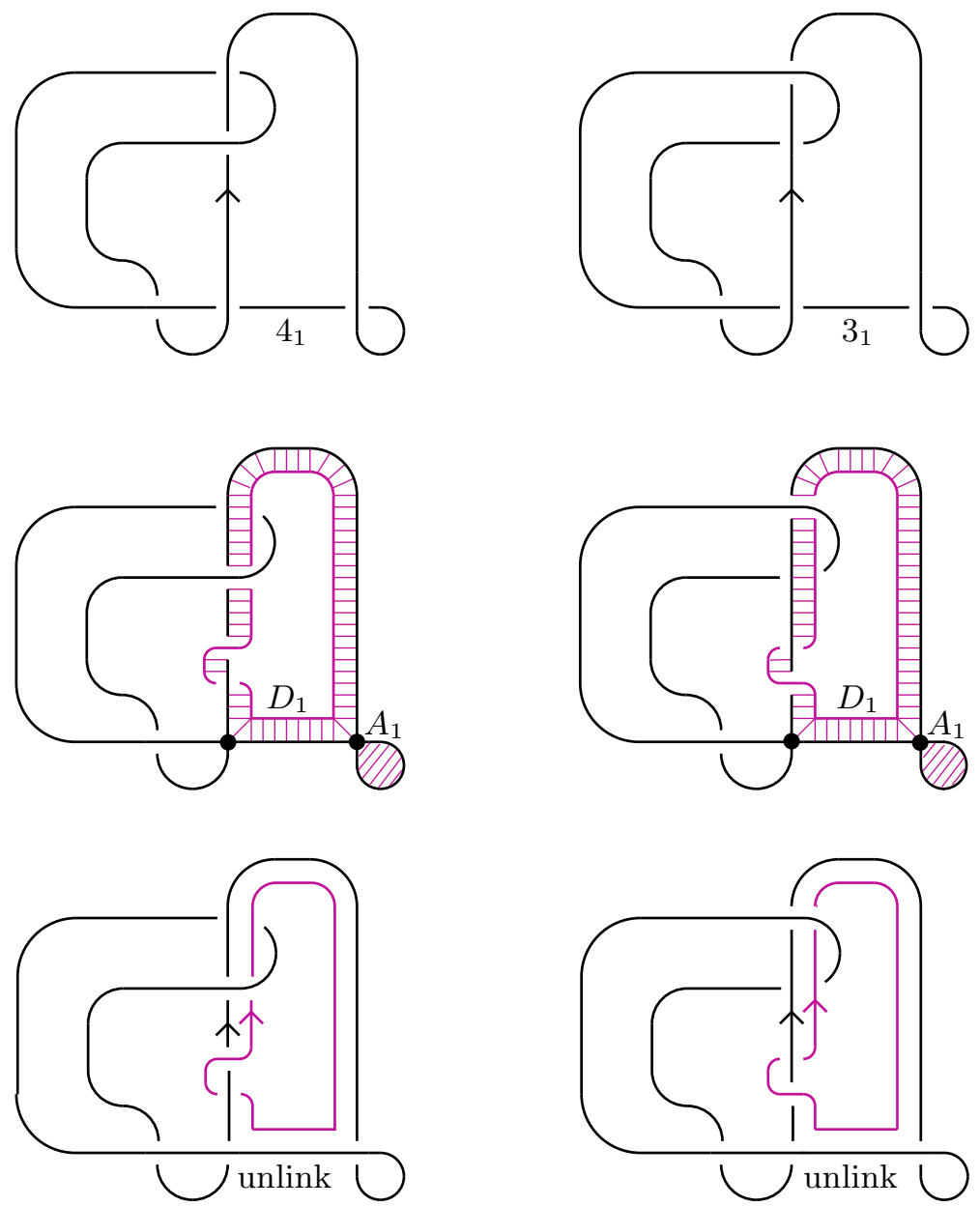

Fig. 14 Movies for twisted Whitney towers in $S^{3} \times I$. Movies go top to bottom. Left 3 pictures: Whitney tower cobounding the figure eight knot $4_{1} \subset S^{3} \times\{0\}$. Right 3 pictures: Whitney tower cobounding the trefoil $3_{1} \subset S^{3} \times\{0\}$. Top picture: the knot, which will evolve via a homotopy to the unknot over time, tracing out an immersed disc $D_{0}$. Middle picture: double points of the immersion of $D_{0}$, a collar $S^{1} \times I$ of the twisted Whitney disc $D_{1}$, and the accessory disc $A_{1}$. Bottom picture. The interior boundary of the collar, together with the knot after the crossing changes from the double points, form an unlink, which can be capped off by two disjoint discs in $S^{3} \times\{1\}$, to complete $D_{0}$ and $D_{1}$. Cap off $S^{3} \times I$ with a copy of $D^{4}$ to obtain a Whitney tower in $D^{4}$

\section{Examples and an algorithm for computation}

\subsection{Using Whitney towers}

We give some examples of computing the Alexander polynomial using Whitney tower data. Movies depicting twisted order one Whitney towers with boundary knots the figure eight knot $4_{1}$ and the trefoil $3_{1}$ are shown on the left and right of Fig. 14 respectively. The movies are explained in the caption to the figure. 
We will simultaneously discuss both examples, indicating differences between the Whitney towers for $3_{1}$ and $4_{1}$ when they arise. The only difference turns out to be one sign change. It is a straightforward computation to see that $\pi_{1}(W) \cong \mathbb{Z}$. Since there is one Whitney disc and one accessory disc, we have that $H_{2}(W ; \mathbb{Z}[\mathbb{Z}]) \cong \mathbb{Z}[\mathbb{Z}]^{2}$, generated by the spheres $S_{1}$ and $S_{2}$, constructed from the Whitney and the accessory disc respectively, as in Sect. 4.2 i.e. $d=2$.

We apply the formula from Theorem 1.2. The Whitney and accessory discs are disjointly embedded. Therefore we just need to compute the twisting coefficients $a_{1}$ and $b_{1}$. The accessory disc is untwisted, so $b_{1}=0$. The crossing change occurring during the top-to-bottom evolution of the bottom right of each knot diagram, where the accessory disc is found in the middle picture, changes a negative crossing to a positive crossing. It is therefore a positive intersection point, so the self-intersection of $S_{2}$ is 1 . On the other hand, the Whitney disc $D_{1}$ is twisted. The linking number of the boundary of $D_{1}$ with the interior of the collar $S^{1} \times I$ in the middle picture, is +1 for the figure eight knot,and -1 for the trefoil. Therefore the twisting of the Whitney framing relative to the disc framing is $a_{1}=-1$ for the figure eight and $a_{1}=+1$ for the trefoil. This yields the following intersection matrices $\Omega$, using the formulae given in the bullet points in Sect. 3. Recall that $z=(1-t)\left(1-t^{-1}\right)=2-t-t^{-1}$. For $4_{1}$, we have

$$
\Omega=\left[\begin{array}{cc}
-z & 1 \\
1 & 1
\end{array}\right],
$$

whose determinant is $-z-1=t+t^{-1}-2-1=t+t^{-1}-3 \doteq \Delta_{4_{1}}(t)$. For $3_{1}$, we have the matrix

$$
\Omega=\left[\begin{array}{ll}
z & 1 \\
1 & 1
\end{array}\right],
$$

whose determinant is $z-1=2-t-t^{-1}-1=1-t-t^{-1} \doteq \Delta_{3_{1}}(t)$.

\subsection{An algorithm for computation using accessory discs only}

By using a natural choice of accessory discs, described below, the computation of the intersection data (and consequently the abelian invariants) can be formulated as an algorithm, that we now describe.

- Fix a given set of crossing changes on a planar diagram of $K$ which convert $K$ to a trivial knot; recall that such a set of crossings can be found on any knot diagram.

- Consider the planar diagram obtained by replacing all the crossings to be changed with a singularity. This is the diagram at the level of the intersection points in a movie picture of the immersed disc in $D^{4}$ arising as the trace of a homotopy realising the crossing changes. The sign of the crossing change determines the sign of the intersection point of $D_{0}$. For each intersection in the singular diagram, draw a double point loop which leaves the crossing, follows along the knot agreeing with the given orientation, leaving along one strand and returning to the crossing along the other strand.

- Push the loop slightly off the singular knot, and twist the loop around the singular knot until the linking number with the singular knot is zero i.e. after the crossing change the linking number with the resulting unknot is 0 .

- Choose basing paths for each accessory loop.

- Now, replace the singular crossings with the outcome of each of the crossings changes, and apply an ambient isotopy which takes the resulting unknot to the standard unknot $U$. 
- Under the isotopy, the union of double point loops becomes an oriented based link, say $L$. The $i$ th component of $L$ will give rise to the $i$ th accessory disc $A_{i}$.

- In the complement of $U$, apply a homotopy of $L$, that is, crossing changes of $L$, dragging the basing paths along, until $L \cup U$ becomes the trivial link. Here crossing changes involving different components of $L$ are allowed. For each crossing change on $L$, record the sign of the crossing change and the element $\ell \in \mathbb{Z}=\pi_{1}\left(S^{3} \backslash \nu U\right)$ determined by linking with $U$ of the usual concatenation of paths in $L$ with the basing paths.

- The number of twists of $L_{i}$ that we made away from the blackboard framing, plus twice the signed count of self intersections of $L_{i}$, determines the negative of the twisting of $A_{i}$. With these considerations the intersection data can be completely recovered.

\subsection{Examples using accessory discs only}

Here is a detailed example of the above algorithm. Consider $K=\mathrm{Wh}_{n}^{-}(J)$, the negatively clasped $n$-twisted Whitehead double of a knot $J$. Here negatively clasped means the signs of the crossings are negative, and $n$-twisted means $n$ full right handed twists; a negative right handed twist, which appears if $n<0$, is a left handed twist. We can change a single crossing from a negative to a positive crossing in the clasp to make a homotopy to the unknot $U$. Therefore $d=1$ and $\varepsilon_{1}=1$. The double point loop becomes a copy of the knot $J$, twisted $-n$ times around this unknot. Add $n$ twists to the double point loop so that it is null homotopic in the complement of $U$. The null homotopy of $J$ produces the accessory disc $A_{1}$. Every double point of $A_{1}$ has the trivial element of $\pi_{1}\left(S^{3} \backslash \nu U\right) \cong \mathbb{Z}$ associated to it. Add local cusps of the appropriate sign so that the signed count of double points of $A_{1}$ vanishes. The matrix $\Psi$ is then a $1 \times 1$ matrix with entry $1+z b_{1}$, where $b_{1}$ is the twisting coefficient. Since we added $n$ positive twists to the double point loop, the twisting coefficient is $-n$, and we compute:

$$
\Delta_{K}(t) \doteq \operatorname{det}(\Psi)=1-n z=1-n\left(2-t-t^{-1}\right)=1-2 n+n t+n t^{-1} .
$$

\section{The Seifert form and the Arf invariant}

We are about to investigate the implications of Theorem 1.2 for the Arf invariant of a knot. First, in this section, we briefly recall the usual definition of the Arf invariant of a knot in terms of a Seifert form.

Definition 8.1 A quadratic enhancement of a symmetric bilinear form $\lambda: M \times M \rightarrow \mathbb{Z}_{2}$ on a $\mathbb{Z}_{2}$ vector space $M$ is a function $q: M \rightarrow \mathbb{Z}_{2}$ such that

$$
q(x)+q(y)+q(x+y) \equiv \lambda(x, y) \bmod 2
$$

for all $x, y \in M$. A quadratic form is a symmetric bilinear form $M, \lambda$ together with a quadratic enhancement $q$.

Let $\left\{e_{1}, f_{1}, e_{2}, f_{2}, \ldots, e_{n}, f_{n}\right\}$ be a symplectic basis for $M$ i.e. $\lambda\left(e_{i}, e_{j}\right)=0, \lambda\left(f_{i}, f_{j}\right)=$ 0 and $\lambda\left(e_{i}, f_{j}\right)=\delta_{i j}$ for all $i, j=1, \ldots, n$. Then the Arf invariant of the quadratic form is

$$
\operatorname{Arf}(M, \lambda, q):=\sum_{i=1}^{n} q\left(e_{i}\right) q\left(f_{i}\right) \bmod 2 .
$$

See [19, Appendix] for the proof that this is well-defined. 
Definition 8.2 We will define a $\mathbb{Z}_{2}$-valued quadratic enhancement on the $\mathbb{Z}_{2}$-valued intersection form on the first homology of a Seifert surface $F$ of the knot. Represent an element of $H_{1}\left(F ; \mathbb{Z}_{2}\right)$ by an oriented simple closed curve $\gamma \subset F$, and define a framing of its normal bundle by choosing a framing of the normal bundle $v_{F \subset S^{3}}$ of $F$ in $S^{3}$. (Using the orientation of $S^{3}$ and $\gamma$ this choice determines a framing of the normal bundle $v_{\gamma \subset F}$, and therefore a framing of $v_{\gamma \subset S^{3}}$ in the conventional sense). Every simple closed curve in $S^{3}$ bounds a closed oriented Seifert surface $G_{\gamma}$, and the unique (up to homotopy) framing of $v_{\gamma \subset S^{3}}$ which extends to a framing of the normal bundle of $G_{\gamma}$ is the zero framing of $\gamma$. We may therefore compare the zero framing of $\gamma$ with the framing defined above by the embedding of $F$, to obtain an integer. This measures the number of full twists in the "band" of the Seifert surface with core $\gamma$. The modulo 2 reduction of this integer defines a quadratic enhancement of the $\mathbb{Z}_{2}$-intersection form on $H_{1}\left(F ; \mathbb{Z}_{2}\right)$, as promised, that is a function $q: H_{1}\left(F ; \mathbb{Z}_{2}\right) \rightarrow \mathbb{Z}_{2}$. The Arf invariant of $K$ is $\operatorname{Arf}\left(H_{1}\left(F ; \mathbb{Z}_{2}\right), \lambda, q\right)$.

\section{Proof of Arf Invariant Theorem 1.9}

Proof of Theorem 1.9 We saw in the proof of Theorem 1.2 that $\operatorname{det}(\Omega(t))=\Delta_{K}(t) f(t) f\left(t^{-1}\right)$ for some $f \in \mathbb{Z}\left[t, t^{-1}\right]$ with $f(1)= \pm 1$. Thus $\operatorname{det}(\Omega(-1))=\Delta_{K}(-1) f(-1)^{2}$. But $f(1)= \pm 1$ implies that $f(-1)$ is odd. Thus $f(-1)^{2} \equiv \pm 1 \bmod 8$, and so we have that $\Delta_{K}(-1) \equiv \operatorname{det}(\Omega(-1)) \bmod 8$. Then observe that $\Omega(t)=\lambda(t) / z$, so $\Omega(-1)=\lambda(-1) / 4$. The form of $\lambda$ in Theorem 3.1 implies that $\Omega(-1)$ reduces to the matrix $A$ in Lemma 9.1 below, with $X(-1)=B, Y(-1)=C, x_{i}=4 a_{i}$ and $y_{i}=4 b_{i}$.

Lemma 9.1 Let $A$ be ad $\times d$ matrix over $\mathbb{Z}$, with $d=2 k$, of the form $B+4 C+4 C^{T}$ where $C$ is upper triangular and $B$ is a block diagonal sum of $2 \times 2$ matrices $D_{i}$ of the form $\left[\begin{array}{cc}x_{i} & 1 \\ 1 & 1+y_{i}\end{array}\right]$, where $x_{i}$ and $y_{i}$ are both a multiple of 4 . Then $\operatorname{det} A \equiv(-1)^{k}+\sum_{i=1}^{k} x_{i} \bmod 8$.

In particular,

$$
(-1)^{k}+\sum_{i=1}^{k} 4 a_{i} \equiv\left\{\begin{array}{lll} 
\pm 1 & \text { if } \sum_{i=1}^{k} a_{i} \equiv 0 & \bmod 2 \\
\pm 3 & \text { if } \sum_{i=1}^{k} a_{i} \equiv 1 & \bmod 2
\end{array}\right.
$$

The count on the right hand side is exactly the number of twisted Whitney discs modulo two. This completes the proof of Theorem 1.9 modulo the proof of Lemma 9.1.

The idea for Lemma 9.1 and its proof come from [14, Section 3.5]. The argument in this lemma is slightly simpler since the contributions from the accessory discs are always odd, thus the Whitney disc terms decide the outcome modulo 8. In the Seifert surface case considered by Levine, the twisting of both of a dual pair of generators determine whether that dual pair contributes to the Arf invariant.

Proof of Lemma 9.1 Following Levine, we call an element of the matrix A special if it is odd: these are the entries $a_{(2 i-1),(2 i)}=a_{(2 i),(2 i-1)}$ and $a_{(2 i),(2 i)}$, for $i=1, \ldots, k$. The remaining entries of $A$ are even and these are called non-special.

The determinant is computed as a sum of terms, where each term is a product of elements, one taken from each row and each column. Note that all the non-special terms are in fact a multiple of 4 . Thus in order for a summand of the determinant to contribute to the reduction modulo 8 it must be a product of elements, at most one of which is non-special. 
We therefore need to look at the summand containing only special terms (there is precisely one such summand) and the summands containing precisely one non-special term. The only summand of the determinant which contains only special terms is

$$
\prod_{i=1}^{k}-a_{(2 i-1),(2 i)} a_{(2 i),(2 i-1)}=(-1)^{k} \prod_{i=1}^{k} a_{(2 i-1),(2 i)}^{2} .
$$

Since $a_{(2 i-1),(2 i)}=1+4 n_{i}$ for some $n_{i} \in \mathbb{Z}$, we have that $a_{(2 i-1),(2 i)}^{2} \equiv 1 \bmod 8$ so that modulo 8 the contribution is $(-1)^{k}$.

There is a summand with precisely one non-special term for each $i=1, \ldots, k$, of the form:

$$
\begin{aligned}
& a_{(2 i-1),(2 i-1)} a_{(2 i),(2 i)} \prod_{1 \leq j<i, i<j \leq k}-a_{(2 j-1),(2 j)} a_{(2 j),(2 j-1)} \\
& =(-1)^{k-1} a_{(2 i-1),(2 i-1)} a_{(2 i),(2 i)} \prod_{1 \leq j<i, i<j \leq k} a_{(2 j-1),(2 j)}^{2} .
\end{aligned}
$$

Let $\alpha_{i}$ and $\beta_{i}$ be such that $x_{i}=4 \alpha_{i}$ and $y_{i}=4 \beta_{i}$. We also may write $a_{(2 i-1),(2 i-1)}=$ $4 \alpha_{i}+8 m_{i}, a_{(2 i),(2 i)}=1+4 \beta_{i}+8 \ell_{i}$ and finally $a_{(2 j-1),(2 j)}=1+4 n_{j}$ as above. Thus modulo 8 we have that each summand

$$
(-1)^{k-1} a_{(2 i-1),(2 i-1)} a_{(2 i),(2 i)} \prod_{1 \leq j<i, i<j \leq k} a_{(2 j-1),(2 j)}^{2} \equiv 4 \alpha_{i} \quad \bmod 8 .
$$

Combining the contributions to the determinant of the summand with all special terms and the $k$ summands with precisely one non-special term, we have that $\operatorname{det} A=(-1)^{k}+\sum_{i=1}^{k} x_{i}$ as claimed.

\section{The Blanchfield form}

In this section we show that the matrices $\Omega$ and $\Psi$ present a linking form in the Witt class of the Blanchfield form of $K$, and that the form they present is isometric to the Blanchfield form of $K$ in the case that the immersed disc $D_{0}$ arises from crossing changes on $K$. This will prove the Blanchfield form statements of Theorems 1.2, 1.3 and 1.4.

Let $R=\mathbb{Z}[\mathbb{Z}$, and let $Q=\mathbb{Q}(\mathbb{Z})$ be its quotient field. A linking form is defined to be a sesquilinear, hermitian, nonsingular form $\beta: V \times V \rightarrow Q / R$ with $V$ a finitely generated torsion $R$-module. Suppose $M$ is a 3 -manifold over $\mathbb{Z}$, that is, $M$ is endowed with a homomorphism $\pi_{1}(M) \rightarrow \mathbb{Z}$. Suppose $H_{1}(M ; R)$ is torsion over $R$ and the map $H_{1}(\partial M ; R) \rightarrow H_{1}(M ; R)$ is the zero map. Then the Blanchfield form [1] of $M$ is defined to be the linking form

$$
\mathrm{B}^{‘}: H_{1}(M ; R) \times H_{1}(M ; R) \longrightarrow Q / R,
$$

whose adjoint $\mathrm{B}^{\star *}$ coincides with the composition of isomorphisms

$$
\begin{aligned}
& H_{1}(M ; R) \longrightarrow H_{1}(M, \partial M ; R) \longrightarrow H^{2}(M ; R) \\
& \quad \longrightarrow H^{1}(M ; Q / R) \longrightarrow \overline{\operatorname{Hom}_{R}\left(H_{1}(M ; R), Q / R\right)} .
\end{aligned}
$$

That is, $\mathrm{B}^{* *}(y)(x)=\mathrm{B}^{\star}(x, y)$. Here the bar denotes the use of the involution on $R$ to convert from a right module to a left module. The morphisms above are given by the long exact 
sequence of the pair $(M, \partial M)$, Poincaré duality, a Bockstein homomorphism and universal coefficients, respectively. The proof that they are all isomorphisms can be found in [15].

The Blanchfield form of an oriented knot $K$ is defined to be that of the exterior $X_{K}$ endowed with the homomorphism $\pi_{1}(M) \rightarrow \mathbb{Z}$ that sends a positive meridian to the generator $t$.

Definition 10.1 We say that an $n \times n$ hermitian matrix $A=A(t)$ over $R$ presents a linking form $\beta$ if $\beta$ is isometric to the sesquilinear pairing

$$
\left(R^{n} / A \cdot R^{n}\right) \times\left(R^{n} / A \cdot R^{n}\right) \longrightarrow Q / R
$$

given by $([x],[y]) \mapsto-y^{T} \cdot \bar{A}^{-1} \cdot \bar{x}$.

Lemma 10.2 Suppose $W$ is a 4-manifold with $\pi_{1}(W)=\mathbb{Z}$ so that $\partial W=M$ is over $\mathbb{Z}$, and $\Lambda=\Lambda(t)$ is a matrix representing the $R$-valued intersection form on $H_{2}(W ; R)$. Then $\Lambda$ presents the Blanchfield form of $M$.

Proof First, $H_{1}(W ; R)=0$ since $\pi_{1}(W)=\mathbb{Z}$. In addition, $H_{2}(W ; R)$ and $H_{2}(W, M ; R)$ are free $R$-modules of the same rank, by Lemmas 4.1 and 6.1. Since $\Lambda$ represents $H_{2}(W ; R) \rightarrow$ $H_{2}(W, M ; R)$, it follows that $\Lambda$ is a presentation matrix for $H_{1}(M ; R)$. Here we fix an arbitrary basis for $H_{2}(W ; R)$ and use the dual basis for $H_{2}(W, M ; R)$ as usual.

Let $\Phi$ be the composition

$$
\begin{aligned}
& \Phi: H_{2}(W, M ; R) \longrightarrow H_{2}(W, M ; Q) \stackrel{\cong}{\rightrightarrows} H_{2}(W ; Q) \longrightarrow H_{2}(W ; Q / R) \\
& \stackrel{\cong}{\longrightarrow} H^{2}(W, M ; Q / R) \longrightarrow \overline{\operatorname{Hom}_{R}\left(H_{2}(W, M ; R), Q / R\right)} .
\end{aligned}
$$

It is known that $\mathrm{B}^{\text {' }}(\partial x, \partial y)=-\Phi(x)(y)$ for any $x, y \in H_{2}(W, M ; R)$; see for instance [4, Lemma 3.3]. (Our sign convention is opposite to that of [4].)

Using bases for $H_{2}(W ;-)$ induced by our fixed basis and using the dual basis for $\mathrm{H}_{2}(W, M ;-)$ once again, all the arrows but the second in the above definition of $\Phi$ are represented by the identity matrix. The second arrow is the inverse of the intersection pairing, and thus represented by $\Lambda^{-1}$. It follows that

$$
\mathrm{B}^{`}(\partial x, \partial y)=-y^{T} \cdot \overline{\Lambda^{-1} x}=-y^{T} \cdot \bar{\Lambda}^{-1} \cdot \bar{x} .
$$

Now consider the case of $W=D^{4} \backslash v D_{0}$. Construct the following commutative diagram, as explained below the diagram:

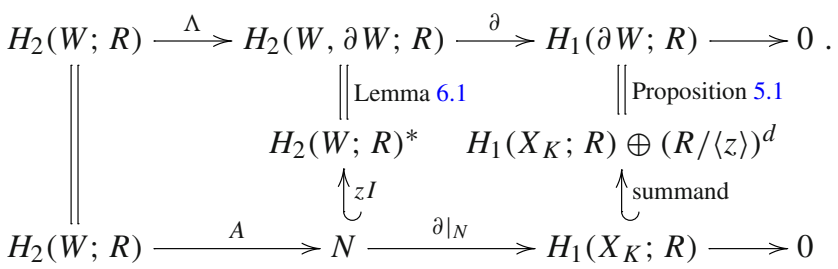

The top row is a part of the long exact sequence for $(W, \partial W)$. Let $N:=z \cdot H_{2}(W ; R)^{*}$. Since $H_{2}(W ; R)^{*} \cong R^{d}, N \cong R^{d}$. Since $\partial(N) \subset z \cdot H_{1}(\partial W ; R) \subset H_{1}\left(X_{K} ; R\right), \partial$ induces $\left.\partial\right|_{N}: N \rightarrow H_{1}\left(X_{K} ; R\right)$. Since $1-t$ is an automorphism on $H_{1}\left(X_{K} ; R\right)$, so is $z=(1-t)(1-$ $\left.t^{-1}\right)$. It follows that $\left.\partial\right|_{N}$ is surjective. Also, since $H_{2}(W ; R)^{*} / N \cong(R /\langle z\rangle)^{d}$, the image of $\Lambda$ lies in $N$. So there is $A: H_{2}(W ; R) \rightarrow N$ making the diagram commute. Multiply our (dual) basis for $H_{2}(W ; R)^{*}$ by $z$ to obtain a basis for $N$. With respect to this, the inclusion $N \rightarrow H_{2}(W ; R)^{*}$ is (represented by) the diagonal matrix $z I$. So $\Lambda=z A$ as matrices. 
Claim The matrix A presents the Blanchfield form $\mathrm{B}^{\text {' }} \mathrm{X}_{K}$ of $K$.

To prove the claim, first observe that the Blanchfield form $\mathrm{B}^{`} \partial W$ of $\partial W$ is given by

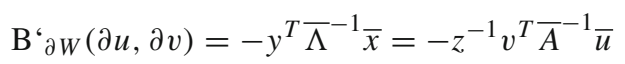

for $u, v \in H_{2}(W ; R)^{*}$, by Lemma 10.2. Using that the bottom row of the above diagram is exact, identify $H_{1}\left(X_{K} ; R\right)$ with $R^{d} / A \cdot R^{d}=N / \operatorname{Im}\{A\}$. Then, from the above description of $\mathrm{B}^{\prime}{ }_{\partial W}$, it follows that

$$
\mathrm{B}^{{ }^{\prime}}{ }_{K}:\left(R^{d} / A \cdot R^{d}\right) \times\left(R^{d} / A \cdot R^{d}\right) \longrightarrow Q / R
$$

is given by $(x, y) \mapsto \mathrm{B}^{{ }}{ }_{\partial W}(x, y)=-y^{T}\left(z \bar{A}^{-1}\right) \bar{x}$. Since $z=(1-t)\left(1-t^{-1}\right)$, it is straightforward to see that the following diagram is commutative:

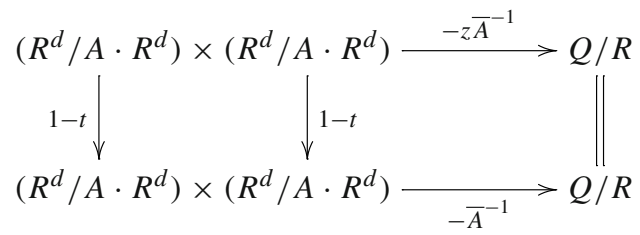

Since $1-t$ is an automorphism on $R^{d} / A \cdot R^{d}=H_{1}\left(X_{K} ; R\right)$, it follows that $A$ presents $\mathrm{B}^{\text {' }} X_{K}$, as claimed above.

In the case that the submodule $F$ generated by our 2 -spheres is equal to $H_{2}(W ; R)$, for example in the special case of an immersed disc arising from crossing changes on $K$, we have $\Lambda=\lambda=z \Psi$, that is, $A=\Psi$. This completes the proof of the Blanchfield form assertion of Theorem 1.4.

In general, namely when $F$ is not necessarily $H_{2}(W ; R)$, let $P=P(t)$ be the square matrix representing the inclusion $R^{d} \cong F \rightarrow H_{2}(W ; R) \cong R^{d}$. The matrix $P(1)$ is unimodular over $\mathbb{Z}$, since our spherical basis elements of $F$ descend to a basis of $H_{2}(W ; \mathbb{Z})$.

Construct the following commutative diagram, as explained below:

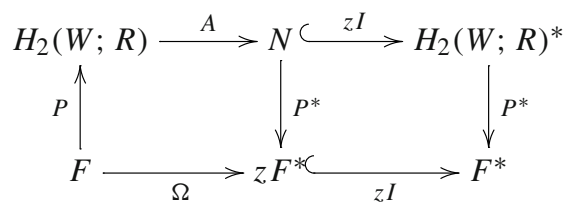

First, choosing the natural basis for $z F^{*} \subset F^{*}$ as we did for $N \subset H_{2}(W ; R)^{*}$, the inclusion $z F^{*} \hookrightarrow F^{*}$ is the diagonal matrix $z I$. Since $F \rightarrow F^{*}$ is the intersection matrix $\lambda=z \Omega$ (or $z \Psi$ ), the map $F \rightarrow z F^{*}$ is represented by the matrix $\Omega$ as in the above diagram. Since $P^{*}$ is $R$-linear, it takes $N=z \cdot H_{2}(W ; R)^{*}$ to $z F^{*}$, namely $P^{*}$ induces the middle vertical arrow in the above diagram. Furthermore, with respect to our basis for $z F^{*}$, the induced homomorphism $N \rightarrow z F^{*}$ is represented by the same matrix $P^{*}$.

From the above diagram, it follows that $\Omega=P A P^{*}$. By the following lemma, $\Omega$ presents a linking form which is Witt equivalent to the Blanchfield form of $X_{K}$. This completes the proof of the Blanchfield form assertions of Theorems 1.2 and 1.3.

Lemma 10.3 (Ranicki) The two linking forms presented by hermitian matrices $A(t)$ and $P(t) A(t) P\left(t^{-1}\right)^{T}$ are Witt equivalent, where $\operatorname{det} P(1)= \pm 1$ and $\operatorname{det} A(t) \neq 0$. 
Proof This lemma appears on Ranicki [18, p. 268], in the proof of his Proposition 3.4.6 (ii). To make the translation from Ranicki's notation to ours without having to read too much of [18], one needs to know that the boundary of a form is the linking form presented by a matrix representing that form, and the fact that $\operatorname{det} P(1)= \pm 1$ implies that $P$ is an isomorphism over $\mathbb{Q}(\mathbb{Z})$, that is $P$ corresponds to an $S$-isomorphism, with $S$ the nonzero polynomials in $\mathbb{Z}[\mathbb{Z}]$.

Acknowledgements We would like to thank Peter Teichner for several useful suggestions, and in particular for sharing the idea of the construction of spheres from Whitney discs. We also thank Nick Castro, Stefan Friedl, Daniel Kasprowski, Allison Moore and Rob Schneiderman for their interest and helpful comments. The second author gratefully acknowledges the support provided by the SFB 1085 Higher Invariants at the University of Regensburg while on sabbatical in the fall of 2014, and funded by the Deutsche Forschungsgemeinschaft (DFG).

Open Access This article is distributed under the terms of the Creative Commons Attribution 4.0 International License (http://creativecommons.org/licenses/by/4.0/), which permits unrestricted use, distribution, and reproduction in any medium, provided you give appropriate credit to the original author(s) and the source, provide a link to the Creative Commons license, and indicate if changes were made.

\section{References}

1. Blanchfield, R.C.: Intersection theory of manifolds with operators with applications to knot theory. Ann. Math. 2(65), 340-356 (1957)

2. Borodzik, M., Friedl, S.: On the algebraic unknotting number. Trans. Lond. Math. Soc. 1(1), 57-84 (2014)

3. Casson, A.J.: Three lectures on new-infinite constructions in 4-dimensional manifolds. In: À la recherche de la topologie perdue, vol. 62 of Progr. Math., pp. 201-244. Birkhäuser Boston, Boston (1986) (With an appendix by L. Siebenmann)

4. Cha, J.C.: Topological minimal genus and $L^{2}$-signatures. Algebraic Geom. Topol. 8, 885-909 (2008)

5. Cochran, T.D., Orr, K.E., Teichner, P.: Knot concordance, Whitney towers and $L^{2}$-signatures. Ann. Math. (2) 157(2), 433-519 (2003)

6. Conant, J., Schneiderman, R., Teichner, P.: Whitney tower concordance of classical links. Geom. Topol. 16(3), 1419-1479 (2012)

7. Conant, J., Schneiderman, R., Teichner, P.: Milnor invariants and twisted Whitney towers. J. Topol. 7(1), 187-224 (2014)

8. Freedman, M., Kirby, R.: A geometric proof of Rochlin's theorem. In: Algebraic and geometric topology (Proc. Sympos. Pure Math., Stanford Univ., Stanford, Calif., 1976), Part 2, Proc. Sympos. Pure Math., XXXII, pp. 85-97. Amer. Math. Soc., Providence, RI (1978)

9. Freedman, M.H., Quinn, F.: Topology of 4-Manifolds. Princeton Mathematical Series, vol. 39. Princeton University Press, Princeton (1990)

10. Friedl, S., Powell, M.: A calculation of Blanchfield pairings of 3-manifolds and knots. arXiv:1512.04603v2 (2016)

11. Kawauchi, A.: Three dualities on the integral homology of infinite cyclic coverings of manifolds. Osaka J. Math. 23(3), 633-651 (1986)

12. Kearton, C.: Blanchfield duality and simple knots. Trans. Am. Math. Soc. 202, 141-160 (1975)

13. Kirby, R.C.: The Topology of 4-Manifolds. Lecture Notes in Mathematics, vol. 1374. Springer, Berlin (1989)

14. Levine, J.P.: Polynomial invariants of knots of codimension two. Ann. Math. 2(84), 537-554 (1966)

15. Levine, J.P.: Knot modules. Trans. Am. Math. Soc. 229, 1-50 (1977)

16. Lightfoot, A.: The Schneiderman-Teichner invariant applied to immersions arising from link maps in $S^{4}$. arXiv:1312.1936 (2013)

17. Matsumoto, Y.: Secondary intersectional properties of 4-manifolds and Whitney's trick. In: Algebraic and geometric topology (Proc. Sympos. Pure Math., Stanford Univ., Stanford, Calif., 1976), Part 2, Proc. Sympos. Pure Math., XXXII, pp. 99-107. Amer. Math. Soc., Providence, RI (1978)

18. Ranicki, A.: Exact Sequences in the Algebraic Theory of Surgery. Mathematical Notes, vol. 26. Princeton University Press, Princeton, N.J. (1981)

19. Rourke, C.P., Sullivan, D.: On the Kervaire obstruction. Ann. Math. 2(94), 397-413 (1971) 
20. Schneiderman, R.: Simple Whitney towers, half-gropes and the Arf invariant of a knot. Pac. J. Math. 222(1), 169-184 (2005)

21. Schneiderman, R.: Whitney towers and gropes in 4-manifolds. Trans. Am. Math. Soc. 358(10), 4251-4278 (2006). (electronic)

22. Scorpan, A.: The Wild World of 4-Manifolds. American Mathematical Society, Providence (2005)

23. Seifert, H.: Über das Geschlecht von Knoten. Math. Ann. 110(1), 571-592 (1935)

24. Terence, C., Wall, C.: Surgery on Compact Manifolds. Mathematical Surveys and Monographs, vol. 69, 2nd edn. American Mathematical Society, Providence, RI (1999) (Edited and with a foreword by A. A. Ranicki)

25. Weibel, C.A.: An Introduction to Homological Algebra. Cambridge Studies in Advanced Mathematics, vol. 38. Cambridge University Press, Cambridge (1994)

Publisher's Note Springer Nature remains neutral with regard to jurisdictional claims in published maps and institutional affiliations. 